NBER WORKING PAPER SERIES

\title{
OBEDIENCE IN THE LABOR MARKET AND SOCIAL MOBILITY: A SOCIO-ECONOMIC APPROACH
}

\author{
Daron Acemoglu \\ Working Paper 29125 \\ http://www.nber.org/papers/w29125 \\ NATIONAL BUREAU OF ECONOMIC RESEARCH \\ 1050 Massachusetts Avenue \\ Cambridge, MA 02138 \\ August 2021
}

Prepared for the Economica Centenary Conference. I thank participants at the conference and Jim Robinson for comments and suggestions. I am grateful to Lauren Fahey, Hyunjin Kim, Carlos Molina, Pascual Restrepo and John Sturm for spectacular research assistance. The views expressed herein are those of the author and do not necessarily reflect the views of the National Bureau of Economic Research.

NBER working papers are circulated for discussion and comment purposes. They have not been peer-reviewed or been subject to the review by the NBER Board of Directors that accompanies official NBER publications.

(C) 2021 by Daron Acemoglu. All rights reserved. Short sections of text, not to exceed two paragraphs, may be quoted without explicit permission provided that full credit, including () notice, is given to the source. 
Obedience in the Labor Market and Social Mobility: A Socio-Economic Approach

Daron Acemoglu

NBER Working Paper No. 29125

August 2021

JEL No. A13,J31,J62,P16

\begin{abstract}
$\underline{\text { ABSTRACT }}$
This paper presents an analysis of what types of values, especially in regards to obedience vs. independence, families impart to their children and how these values interact with social mobility. In the model, obedience is a useful characteristic for employers, especially when wages are low, because independent workers require more incentives (when wages are high, these incentives are automatic). Hence, in low-wage environments, low-income families will impart values of obedience to their children to prevent disadvantaging them in the labor market. To the extent that independence is useful for entrepreneurial activities, this then depresses their social mobility. High-income and privileged parents, on the other hand, always impart values of independence, since they expect that their children can enter into higher-income entrepreneurial (or managerial) activities thanks to their family resources and privileges. I also discuss how political activity can be hampered when labor market incentives encourage greater obedience and how this can generate multiple steady states with different patterns of social hierarchy and mobility.
\end{abstract}

Daron Acemoglu

Department of Economics, E52-446

Massachusetts Institute of Technology

77 Massachusetts Avenue

Cambridge, MA 02139

and NBER

daron@mit.edu 


\section{Introduction}

There is increasing recognition that social norms, various dimensions of preferences and values imparted by families and communities are linked to economic and political outcomes 1 Although the emphasis of the earlier literature has been on the effects of cultural factors on economic outcomes, many aspects of culture are also likely to be endogenous to economics and politics. $2^{2}$ This paper builds on this "economics of culture" literature, but also departs from it in two ways. First, I build on Acemoglu and Robinson (2021) who proposed a framework in which cultural configurations are highly adaptable ("fluid") and responsive to changes in the balance of political power or economic incentives, because fluid cultures allow their attributes to be rewired to generate several feasible cultural configurations. In line with this perspective, I explore the co-determination of culture with equilibrium in the labor market - which in particular impacts social incentives via the level of labor demand, wages and patterns of employment rationing. Second, differently from Acemoglu and Robinson (2021), my focus is on how very different cultural configurations emerge between different strata of the same society. In particular, the model developed in this paper emphasizes how the labor market equilibrium generates divergent incentives across agents with different social and economic roles. This encourages elites/entrepreneurs to adopt parenting styles and socialization methods that cultivate greater independence among their offspring $]^{3}$ while pushing non-elites/workers towards obedience, which promotes an internalized social hierarchy in society. 4

Social hierarchy, and specifically whether individuals have internalized notions of "obedience" affecting their economic and political behavior, is a basic building block of any social order. Indeed, many theories going back to Durkheim place social hierarchy at the center of a society's culture. The idea that social hierarchy is maintained because "elites" have dominant values, while non-elites have internalized subordinate or obedient values goes back at

\footnotetext{
${ }^{1}$ See, among others, Putnam (1993, 2000), Greif (1994), Roland (2004), Tabellini (2008, 2010), Mokyr (1990, 2016), Guiso, Sapienza and Zingales (2009), Fernandez and Fogli (2009), Algan and Cahuc (2010), Besley and Persson (2019), Besley (2020), Alesina, Giuliano and Nunn (2013) and Giuliano (2007).

${ }^{2}$ Recent work emphasizing the two-way causality between culture (or social norms) and economic or political institutions includes Tabellini (2009), Acemoglu and Jackson (2015), Bisin, Seror and Verdier (2019), Besley and Persson (2019) and Fernandez (2011).

${ }^{3}$ The use of the notion "value" follows Parsons (1951) and is in line with the vast majority of the economics literature on this topic. Nevertheless, I note that this is a major simplification, and in practice, cultural configurations may not determine unique values, as emphasized by Geertz (1973) Swidler (1986) and Sewell (2005) and recently discussed in Acemoglu and Robinson (2021).

${ }^{4}$ In economics, parenting styles have recently been studied by Doepke and Zilibotti (2012, 2019). Independent versus obedient values are closely related to their notion of "authoritative" versus "authoritarian" parenting.
} 

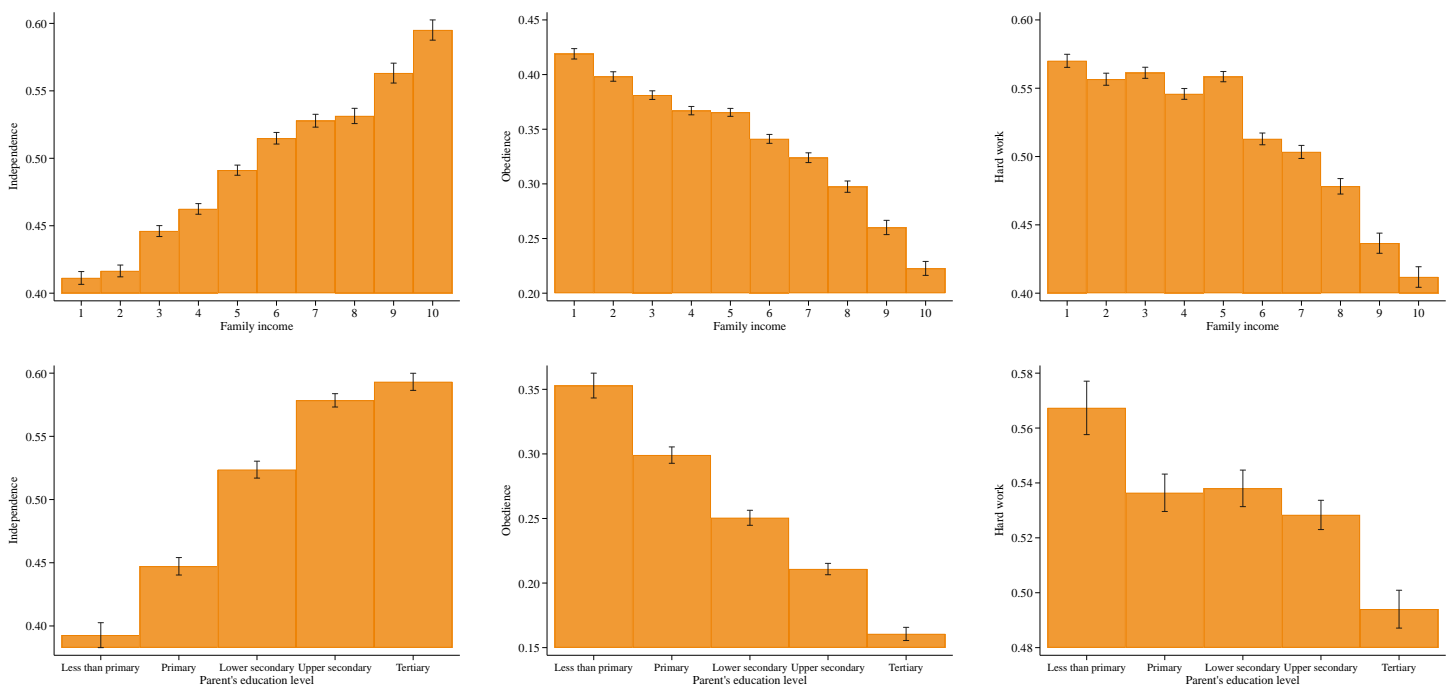

Figure 1: Attitudes towards independence, obedience, and hard work by family income and parents' education, 1981-2018: This figure presents unconditional averages (orange bars) and the two standard-error confidence intervals (black whiskers) of support for independence, obedience and hard work for different levels of family income (top three panels) and parent's education level (bottom three panels) from the Integrated Value Survey for all available waves from 1981 to 2018. See the Appendix for variable definitions and sample information.

least to Inkeles (1960) 55 It is also linked to the notion of "power distance", whereby higher power distance corresponds to a more hierarchical society, with those at the top commanding greater power than the rest, often because of differences in internalized values and attitudes (e.g., Bourdieu and Passeron, 1990, Willis and Willis, 1981, Hofstede, 1980, Oakes, 2005) ${ }^{6}$

Figure 1 uses data from the World Values Survey and shows that, consistent with these ideas, the importance that parents attach to independence, obedience and hard work (which I interpret to be related to obedience) is strongly related to family income and father's education-positively for independence and negatively for obedience and hard work.7 Fig-

\footnotetext{
${ }^{5}$ This type of internalized social hierarchy transmitted within family (or more generally community) is consistent with several lines of work. Campbell et al.'s classic book (1960) emphasized the formation of divergent political preferences within the family (see also Nie, Verba and Petrocik, 1976, Miller, Shanks and Shapiro, 1996, Ventura, 2001, Jennings, Stoker and Bowers, 2009). The system justification theory developed by Jost and collaborators (e.g., Jost and Major, 2001, Haines and Jost, 2000, and Jost, Pelham and Carvalo, 2002) focuses on the internalization of social hierarchy, and the social psychology literature explores the psychological channels via which this may happen (e.g., Miller and Kaiser, 2001). The role of different parenting styles and values by families from varying social economic backgrounds has been emphasized by several scholars, including Kohn (1959, 1989), Lipset (1959) and Pearlin and Kohn (1966) and the literature building on these early works (see also Doepke and Zilibotti, 2012, 2019). The broader role of power relations in workplaces, schools and society at large has been emphasized in the economics literature in Bowles and Gintis (1976).

${ }^{6}$ One of the oft-emphasized cultural differences across different communities and groups is between individualism and collectivism (e.g., Durkheim, 1893; Greif, 1994; Hofstede, 1980). This is closely related to but different from the distinction between independence and obedience. In contrast to individualism vs. collectivism, in the framework here the choice of independence versus obedience is not a uniform one across agents, but rather one in which some social economic groups choose obedience, while others take a more dominant position, often partly by imparting independent values.

${ }^{7}$ The notes to the figure give the exact wording for the relevant questions and sample sizes.
} 


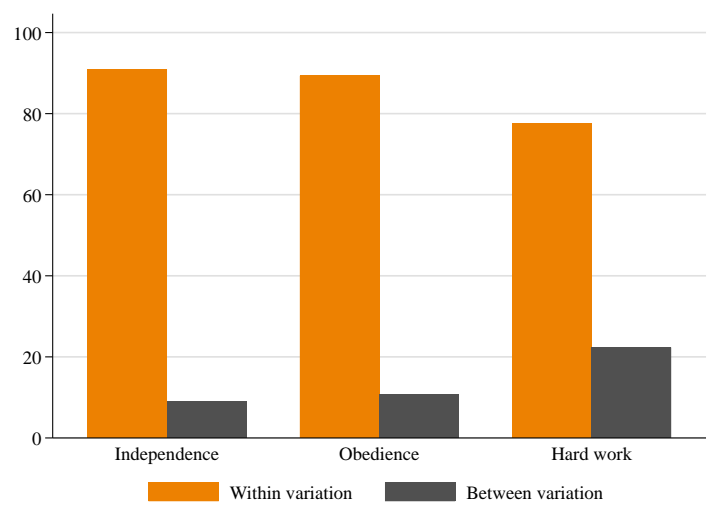

Figure 2: Within and between country variation on attitudes towards independence, obedience, and hard work from Figure 1 .

ure A1 in the Appendix shows an analogous relationship once country, survey and cohort fixed effects are taken out, demonstrating that the same relationship is true within countries and cohorts. In short, rich and educated parents appear to value very different traits - related to independence - in their children than less prosperous and less educated ones.

In line with the emphasis on within-society heterogeneity in cultural configurations, Figure 2 shows that about $90 \%$ of the variance of all three variables plotted in Figure 1 are within country, with the between-country component accounting for not much more than 10\%. Put simply, in contrast to the common emphasis on cross-country differences in values and attitudes, when it comes to independence vs. obedience, most of the variation is within country and accounted for by the social status of parents.

The main question addressed in this paper is how the labor market equilibrium influences obedience vs. independence, providing a potential explanation for the within-country variation shown in Figures 1 and 2

The emphasis on the labor market's effects on culture and values is seldom considered in the economics literature. Nevertheless, many sociology works emphasize the broader social role of obedience in workplaces (e.g., Negandhi and Prasad, 1971, Edwards, 1976, Kohn, 1969, Hofstede, 1980, Willis and Willis, 1981). Closer to economics, Bowles and Gintis (1976) argued that the major problem of capitalist economies is to prepare workers for obedience to their bosses and interpreted the role of schooling in the United States in this light.

This paper builds on these insights, but develops them in a different direction than the previous literature. I assume that workers can be either obedient or independent. Independent workers can become entrepreneurs and are also more productive in regular production tasks (for example, because they generate more problem-solving ideas). In contrast, obedient work- 
ers cannot become entrepreneurs and have lower productivity in production tasks. However, obedient workers are easier for employers to monitor and direct than are independent workers. Specifically, because of standard incentive reasons combined with limited liability, employers have to pay efficiency wages to their workers. Obedient workers are less likely to deviate from the rules imposed on them by their employers, and hence require a lower efficiency wage premium. This may make them more profitable to employ despite their lower productivity.

The key question in the model turns on whether parents prefer to impart obedient or independent values in their children. For parents who know that their child will become an entrepreneur, independence is always preferred. For parents expecting that their children will become a worker with a high probability, there is a trade-off. Independent workers will need to be paid a higher efficiency wage and thus will have greater earning conditional on being employed. On the other hand, if there is insufficient labor demand, then obedient workers who can work "for cheap" (for a smaller efficiency wage premium) will find jobs, while independent workers may be rationed out. This may then motivate parents to go for obedience rather than independence. In summary, when labor demand is limited and employment rationing is low, non-elites will have an incentive for obedience-focused parenting. More generally, this mechanism captures the phenomenon that, in some societies, parenting styles and family decisions can be a potent source of persistence of social hierarchy. For example, the well-off inculcate attitudes to their children that make them feel privileged, which then becomes useful in their professional and social life, while the less well-off inculcate different attitudes, encouraging obedience.

These cultural values may influence the demand side of the labor market via the number and productivity of entrepreneurs in the economy. When non-elites impart obedient values, their children may lack the skills to become entrepreneurs and may further keep labor demand low.

I also outline how obedience-independent values affect political equilibria. This aspect matters in the context of the current model because the political process will be one of the factors affecting entry barriers into entrepreneurship, which then indirectly matters for wage levels, the extent of rationing and preferences for independence versus obedience. The key assumption here is that obedient individuals are also politically less active in defending their (and their economic and social) interests. This opens the way to a multiplicity of steady states: when there is a lot of social mobility, workers expect their children to have economic opportunities and choose independent parenting styles. Independent types are active in politically supporting 
their interests, which keeps entry barriers low and benefits them both directly (they can enter entrepreneurship) and indirectly (wages are higher with lower entry barriers). In contrast, when there is little social mobility, workers expect their children to be economically disadvantaged and choose obedient parenting. This not only keeps entry into entrepreneurship low, but also supports a political equilibrium with high entry barriers, because obedient workers are less likely to vote for or organize in pursuit of their own economic interests. To the best of my knowledge, this is a new source of multiplicity of equilibria, supporting very different types of social mobility and hierarchies.

In addition to the economics of culture and sociology literatures discussed above, this paper is related to a small literature in economics studying social mobility (e.g., Piketty, 1995, Benabou and Ok, 2001, Leventoğlu, 2004, Acemoglu, Egorov and Sonin, 2018). None of these papers explore the cultural foundations of social mobility, even though these cultural ideas are mentioned in qualitative accounts of social mobility, such as Erikson and Goldthorpe (1992).

The rest of the paper is organized as follows. The next section introduces the baseline model, and presents the analysis of this environment without any social mobility and then with endogenous social mobility. Section 3 endogenizes political behavior and contains the result on multiplicity of socio-political equilibria. Section 4 concludes with a brief discussion of various extensions and topics for future research. The Appendix contains various extensions.

\section{Baseline Model: Socio-Economic Equilibrium}

\section{$2.1 \quad$ Environment}

The economy is in discrete time and is populated by a continuum 1 of non-overlapping generations. Each individual lives for one period and begets a single offspring. The main interaction within the family is a parent's choice of "values" or preferences for their offspring. Instantaneous (stage) utilities are defined over expected net income (so that all agents are risk neutral). In particular, individual $h$ of generation $t$ has overall utility

$$
U_{t}^{h}=\xi\left(u_{t}^{h}-B_{t}^{h}\right)+(1-\xi)\left(u_{t+1}^{h}-B_{t+1}^{h}\right)
$$

where $u_{t}^{h}$ denotes this individual's expected income, $B_{t}^{h}$ is a non-pecuniary cost due to potential entry barriers incurred when a non-entrepreneur enters into entrepreneurship, $u_{t+1}^{h}$ and $B_{t+1}^{h}$ denote her offspring's expected income and entry cost, and $\xi \in(0,1)$. This specification imposes a type of "warm glow" altruism where parents care about the net income of their 
offspring, but not about the continuation utility. In addition, in what follows, I focus on the case where $\xi \rightarrow 1$, so that the individual cares much more about her net income than her offspring. This will ensure that current occupation decisions are not made in order to reduce the next generation's entry costs.8

I also assume that there is a small probability $\varepsilon>0$ that a dynasty will come to an end and there is a measure $\varepsilon$ of new dynasties coming in to keep the population constant (and these dynasties start as non-entrepreneurs and are thus subject to entry barriers). This churning of dynasties is introduced as a "selection device" in order to remove some uninteresting indeterminacy (which arises because there are kinks in labor demand due to entry barriers). Since $\varepsilon$ is playing the role of a selection device, in what follows I focus on the case in which $\varepsilon \rightarrow 0$.

Each individual has a type denoted by $\theta_{t}^{h} \in\{o, i\}$ where $o$ designates "obedient" and $i$ corresponds to "independent" ? Throughout an individual's type is public knowledge. How these affect individual preferences and productivity will be discussed below. For now, I specify that each parent $h$ (of generation $t$ ) decides their "parenting style" — obedient or independent - corresponding to their decision of whether to impart obedient or independent values to their offspring. These parenting style decisions are denoted by $v_{t}^{h} \in\{o, i\}$. If a parent of type $\theta$ chooses parenting style $v$, then the type of the offspring will be $\theta_{t+1}^{h}=o$ with probability $1-\pi_{\theta v}$ and $\theta_{t+1}^{h}=i$ with probability $\pi_{\theta v}$. We assume that $\pi_{\theta v}$ satisfies the following two natural conditions:

$$
1>\pi_{i \cdot}>\pi_{o \cdot}>0 \text { and } 1>\pi_{\cdot i}>\pi_{\cdot o}>0
$$

implying that all else equal, parents who are themselves independent are more likely to have independent offspring, parents who are choosing to impart independent values are more likely to have independent offspring, and all of these probabilities are between 0 and 1.10

There are two occupations: entrepreneurship and production work. Individuals who are not in entrepreneurship or production work are unemployed and receive zero income (as an innocuous normalization). In what follows, I will use the index $h^{e}$ for an agent who is an

\footnotetext{
${ }^{8}$ Alternatively, parents may fail to take into account the dynamic implications of their occupational choices due to myopia. If they were to take them into account, this would lead to a dynamic programming problem, which is analyzed in detail in Acemoglu (2008). Doing so would not change any of the qualitative features emphasized here, though the analysis would become more involved.

${ }^{9}$ As discussed in the Introduction, throughout "independent" refers to an individual with greater initiative as well as independence and less willingness to abide by organizational or societal rules.

${ }^{10}$ One could go further and allow these probabilities to also depend on whether a parent is an entrepreneur or a worker. This would add an additional degree of history dependence without affecting the main results emphasized here (but slightly complicating the notation).
} 
entrepreneur and $h^{n}$ for a non-entrepreneur (worker). I will use the index $h$ for a generic parent, when there is no need to distinguish between entrepreneurs and non-entrepreneurs. I will also denote the fraction (measure/number) of independent types among entrepreneurs at time $t$ by $\mu_{t}^{e}$, and the fraction of independent types among non-entrepreneurs at time $t$ by $\mu_{t}^{n}$.

As a production worker, each obedient individual can supply one efficiency unit of labor, while each independent worker can supply $\gamma>1$ efficiency units of labor (i.e., such a worker is $\gamma$ times more productive per hour as an obedient worker). Both types of workers are also subject to a moral hazard problem. In particular, an individual can "shirk" in which case she will not be productive. I assume that a shirking worker receives a non-pecuniary benefit $v>0$ and will be caught with probability $\delta \in(0,1)$. In addition to the difference in productivity resulting from different efficiency units of labor supply, obedient workers, by virtue of their greater willingness to abide by organizational rules, have a lower return to shirking. We model this by assuming that they incur an additional cost $\kappa \in(0, v)$ when they shirk, while independent workers do not. When either type of worker exerts effort (rather than shirking), then every efficiency unit of labor has the same productivity. Finally, I assume that there is limited liability, and thus workers cannot be punished with negative wages.

Let us next derive the incentive compatibility constraints for independent and obedient workers. Since the marginal product of each efficiency unit of labor (conditional on nonshirking) is the same, it is convenient to formulate these constraints in terms of wage per efficiency unit of labor, $w$. Then the incentive compatibility constraint for independent workers is $\gamma w \geq(1-\delta) \gamma w+v$, or

$$
w \geq \underline{w}_{i} \equiv \frac{v}{\delta \gamma}
$$

The left-hand side is the wage the worker will get if he does not shirk. The right-hand side is his expected utility if he shirks: he is not caught and receives the total wage $\gamma w$ ( $\gamma$ units of labor times wage for efficiency unit of labor, $w$ ) with probability $1-\delta$ as well as the utility from shirking $v$, and is caught and just receives the utility from shirking with probability $\delta$. The second line rearranges the incentive compatibility constraint and specifies the minimum wage that an independent worker needs to be paid so that he does not shirk, and defines $\underline{w}_{i}$ as the minimum wage at which an independent individual could be employed as a production worker.

The incentive compatibility constraint for obedient workers is similar except that such a worker has one efficiency unit of labor and incurs the additional cost $\kappa$ if she shirks. This 
implies a similar incentive compatibility constraint for independent workers:

$$
\begin{aligned}
w & \geq(1-\delta) w+v-\kappa \\
& \geq \underline{w}_{o} \equiv \frac{v-\kappa}{\delta} .
\end{aligned}
$$

This equation analogously defines $\underline{w}_{o}$ as the wage at which an obedient individual can be employed as a production worker. The assumption that $\kappa<v$ implies that an obedient worker, working at the minimum wage consistent with non-shirking, $\underline{w}_{o}$, will still receive a positive wage and a rent.

I next impose a crucial assumption for the rest of our analysis:

\section{Assumption 1}

$$
\kappa>\frac{\gamma-1}{\gamma} v
$$

This assumption implies

$$
\underline{w}_{i}>\underline{w}_{o}
$$

so that, even taking into account the greater productivity of independent workers, their moral hazard problem is worse than that of obedient workers. In particular, the wage that will ensure that they do not shirk, $\underline{w}_{i}$, is greater then the incentive compatible wage for obedient workers, $\underline{w}_{o}$. This introduces a key trade-off into our economic environment: from a productive efficiency perspective, it is better for production workers to be independent, but this also necessitates higher wages (for incentive compatibility purposes). For this reason, depending on labor demand, the equilibrium might create an advantage for obedient workers.

The final difference between independent and obedient individuals is in entrepreneurship. Independent individuals are assumed to be more productive as entrepreneurs (because they have greater initiative or creativity). In particular, I assume that an individual of type $\theta$ has access to the following production technology:

$$
\frac{\varkappa}{\alpha} A_{\theta} l_{t}^{\alpha}
$$

(Production function)

where $l_{t}$ denotes the number of production workers hired at time $t, \alpha \in(0,1), A_{i}>A_{o}>0$, and $\varkappa$ is a constant. In what follows, I will set $\varkappa \equiv \alpha^{1+\alpha}(1-\alpha)^{1-\alpha}$ to simplify the expressions.

Since labor markets are competitive, the demand for efficiency units of labor by an active entrepreneur of type $\theta$ when the equilibrium wage is $w_{t}$, denoted by $l_{\theta}\left(w_{t}\right)$, is given by

$$
\varkappa A_{\theta} l_{\theta}\left(w_{t}\right)^{\alpha-1}=w_{t},
$$


or

$$
l_{\theta}\left(w_{t}\right)=\left(\frac{\varkappa A_{\theta}}{w_{t}}\right)^{\frac{1}{1-\alpha}} .
$$

Note that I have already imposed the equilibrium restriction that all efficiency units of labor will trade at the same price $w_{t}$. In particular, there will not be a separate price for the labor hours of obedient and independent workers. This simply follows from competitive labor markets: conditional on incentive compatibility, one efficiency unit of labor from either type of worker has the same productivity, so if one efficiency unit of labor by an independent worker were more expensive, then all employers would prefer to hire only obedient workers, and vice versa. This, however, does not rule out the possibility that only one type of worker will be hired in equilibrium, as I describe next.

Finally, I assume that the entry cost $B_{t}$ is nonpecuniary (meaning that this cost is pure waste). This cost is incurred by the offspring of a non-entrepreneur if she decides to enter entrepreneurship at time $t$. Offspring of entrepreneurs do not incur this cost, because their parent can pass their business to them (similar to Acemoglu, 2008). This description implies that, in terms of equation (1), $B_{t}^{h}=B_{t}$ if dynasty $h$ was not in entrepreneurship at time $t-1$ and chooses to enter entrepreneurship at time $t$, and $B_{t}^{h}=0$ otherwise. $B_{t}$ will be endogenized as part of a political equilibrium later.

\subsection{Definition of Equilibrium}

A socio-economic equilibrium, or equilibrium for short, is defined as a dynamic allocation in which all individuals make the (privately) optimal occupational choices, labor markets clear subject to "incentive compatibility," and all parents impart optimal values to their offspring at each point in time.

More specifically, in a socio-economic equilibrium, each individual $h$ of generation $t$ and of

type $\theta$ and current occupational status $j \in\{e, n\}$ chooses: (i) $v_{t}^{h^{j}}$ to maximize utility, (1) (which amounts to maximizing the net income of their offspring, $u_{t+1}^{h}-B_{t+1}^{h}$ ); (ii) their occupation to maximize their own net income, $u_{t}^{h}-B_{t}^{h}$, given the entry barrier $B_{t}$ and the market wage $w_{t}$; and (iii) if he becomes an entrepreneur, he then chooses a level of employment $l_{\theta}\left(w_{t}\right)$ to maximize profits, i.e., as given by (4). The market wage $w_{t}$ at time $t$ is such that the labor market at time $t$ clears with the following restrictions and rationing rule: (i) the market wage $w_{t}$ is incentive compatible, meaning that all workers employed in production work weakly prefer not to shirk (i.e., if only obedient types will be employed in production work, then 
$w_{t} \geq \underline{w}_{o}$, and if both types will be employed in production work, then $\left.w_{t} \geq \underline{w}_{i}\right)$.11 (ii) if given occupational and labor demand choices, total labor demand is equal to labor supply at some incentive compatible wage $w^{e}$ (i.e., with $w^{e} \geq \underline{w}_{i}$ ), then $w_{t}=w^{e}$ is the equilibrium wage; (iii) if not, then some workers who prefer to be in production work will remain unemployed; (iv) the rationing rule is such that if the market wage satisfies $w_{t} \geq \underline{w}_{i}$ and both obedient and independent types prefer production work, then priority is given to obedient types.

The definition is standard except three features: first, it has a "social" element, given by the choice of values by parents. Second, there is the rationing requirement, which will be explained further below. Third, recall that we are taking $\xi$ in (1) to be arbitrarily close to 1 , so that when making their occupational decisions, individuals do not take into account the implications of these for the potential future entry costs of their offspring (in case they decide to enter entrepreneurship), but their parenting decisions are made to maximize their offspring's net income.

Figure 3 illustrates this definition in simple labor supply-demand diagram and motivates the rationing assumption. Incentive compatible labor supply takes the form of a step function, since obedient workers can be employed only at wages above $\underline{w}_{o}$ (otherwise they will shirk and not be productive), and independent workers can be employed only at wages above $\underline{w}_{i}>\underline{w}_{o}$. Thus if labor demand is limited, as shown in the first panel, the equilibrium will have a wage between $\underline{w}_{o}$ and $\underline{w}_{i}$, and only obedient workers will be employed.

But what happens if labor demand is greater than this amount but still less than what is required for full employment, as shown in the second panel? Now a rationing rule between the two types of workers needs to be specified. If we were to set up the definition of equilibrium as a game-theoretic one, with workers or firms making offers to the other side of the market, then priority in employment would naturally go to obedient types. This is because any allocation in which the wage is equal to $\underline{w}_{i}$ and some obedient types are left unemployed, while some independent types are employed, would lead to a profitable deviation of the following form: a firm would make an offer to an unemployed obedient worker at some wage strictly greater than $\underline{w}_{o}$ and strictly less than $\underline{w}_{i}$ (or the worker would make a similar offer). This deviation would strictly increase the utility of both parties. To rule out such deviations, the equilibrium should give priority to obedient workers whenever there is rationing. To simplify the description of strategies, I use a definition based on competitive equilibrium with rationing and impose this

\footnotetext{
${ }^{11}$ This description makes use of Assumption 1, ensuring that $\underline{w}_{i}>\underline{w}_{o}$, so that if there is rationing, obedient workers will be chosen first.
} 
particular rationing rule. This is without any loss of generality relative to adopting the more elaborate game-theoretic definition of equilibrium.
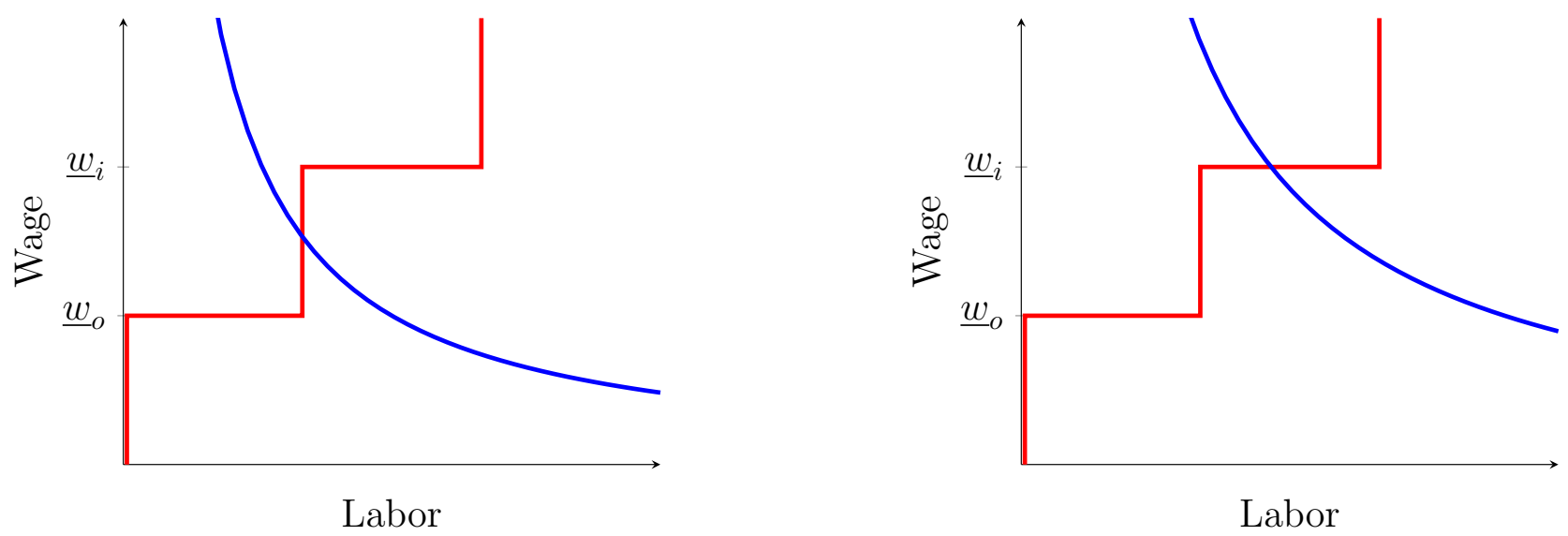

Figure 3: Labor Market Equilibrium with Low Demand and Intermediate Demand

A steady-state (social-economic) equilibrium is simply a (social-economic) equilibrium in which prices and quantities are constant over time.

Finally, throughout I refer to a dynamic or steady-state equilibrium as unique if prices and quantities are uniquely determined.

\subsection{Equilibrium with No Social Mobility}

I first analyze the economy without any social mobility. This implies $B_{t}>>0$ for all $t$ so that it is prohibitively expensive for the offspring of a non-entrepreneur to become an entrepreneur at any point in time. I also assume that there is no exit from entrepreneurship 12

At the wage rate $w$, the profits of an obedient entrepreneur can be written as

$$
\begin{aligned}
\Pi_{\theta}(w) & =\frac{\varkappa}{\alpha} A_{\theta} l_{\theta}(w)^{\alpha}-w l_{\theta}(w) \\
& =w^{-\frac{\alpha}{1-\alpha}} A_{\theta}^{\frac{1}{1-\alpha}}
\end{aligned}
$$

where $l_{\theta}(w)$ is the optimal labor demand of an entrepreneur of type $\theta$ at the wage $w$ given by the expression in (4), and the second line follows by substituting it in and using the normalization

\footnotetext{
${ }^{12}$ The exact expression for the threshold above which the entry barrier needs to be is provided in the next subsection, where I also provide conditions under which no entrepreneur would like to exit.
} 
introduced above, $\varkappa \equiv \alpha^{1+\alpha}(1-\alpha)^{1-\alpha}$. For an obedient individual to prefer entrepreneurship it is sufficient to have $\Pi_{o}\left(\underline{w}_{o}\right)=\underline{w}_{o}^{-\frac{\alpha}{1-\alpha}} A_{o}^{\frac{1}{1-\alpha}} \geq \underline{w}_{o}$, or $A_{o} \geq \underline{w}_{o}$. Independent types are more productive in entrepreneurship, and when employed, will be more productive and earn higher wages in production work as well.In what follows, I impose a sufficient condition to ensure that both obedient and independent types prefer entrepreneurship when the wage is $\underline{w}_{i}$ :

\section{Assumption 2}

$$
A_{o}>\underline{w}_{i} \text { and } A_{i}>\gamma^{1-\alpha} \underline{w}_{i}
$$

Just like Assumption 1, this assumption will be maintained throughout, even when it is not stated.

Armed with these assumptions, we can now characterize the dynamic equilibrium without social mobility. We first determine the parenting styles of entrepreneurs.

Claim 1 In an equilibrium with no social mobility, entrepreneurs will always impart independent values to their offspring, i.e., $v_{t}^{h^{e}}=v_{i}$ for all $t$.

This follows directly from the fact that children of entrepreneurs will be entrepreneurs and independent entrepreneurs have sufficiently high productivity. For this reason, every entrepreneur prefers their offspring to be independent, and since $\pi_{i i}>\pi_{o i}$, every entrepreneur will prefer to adopt an independent parenting style.

Let us next determine the evolution of high-productivity (independent) entrepreneurs starting with initial condition of $E_{0} \in(0,1)$ entrepreneurs with a fraction $\mu_{0}^{e}$ of them being independent. From Claim 1, all entrepreneurs choose to impart independent values to their offspring, and as a result the dynamics of $\mu_{t}^{e}$ without social mobility are given by

$$
\mu_{t+1}^{e}=\pi_{i i} \mu_{t}^{e}+\pi_{o i}\left(1-\mu_{t}^{e}\right)
$$

which follows simply from the fact that the probability that the offspring of an independent entrepreneur (choosing to impart independent values) is independent is $\pi_{i i}$, whereas the same probability for the offspring of a non-entrepreneur (again choosing to impart independent values) is $\pi_{o i}$. It is straightforward to verify that, given (2), $\mu_{t}^{e}$ monotonically converges to a unique steady-state value,

$$
\bar{\mu}^{e}=\frac{\pi_{o i}}{1-\pi_{i i}+\pi_{o i}} .
$$

Let us next turn to labor demand. This follows from the profit maximizing decisions of entrepreneurs of different types - in particular, from the decisions of independent entrepreneurs 
who have productivity $A_{i}$ and of obedient entrepreneurs who have productivity $A_{o}$. Using (4), total labor demand in the no social mobility equilibrium, as a function of the wage, $w_{t}$, can be written as

$$
L_{D}^{\mathrm{NSM}}\left(w_{t} \mid E_{0}, \mu_{t}^{e}\right) \equiv E_{0}\left[\mu_{t}^{e} l_{i}\left(w_{t}\right)+\left(1-\mu_{t}^{e}\right) l_{o}\left(w_{t}\right)\right]=E_{0}\left(\frac{\varkappa}{w_{t}}\right)^{\frac{1}{1-\alpha}}\left[\mu_{t}^{e} A_{i}^{\frac{1}{1-a}}+\left(1-\mu_{t}^{e}\right) A_{o}^{\frac{1}{1-a}}\right] .
$$

Note that the total number of entrepreneurs is constant at $E_{0}$ since there is no social mobility (entry costs are prohibitively high and there is no exit from entrepreneurship). The labor demand schedule evolves over time only because of changes in the fraction of independent entrepreneurs, $\mu_{t}^{e}$.

Equilibrium in the labor market can take one of three forms. To describe this, let us also denote the fraction of independent types among non-entrepreneurs (workers) by $\mu_{t}^{n}$, which will determine the effective units of labor in the population (since independent workers have $\gamma>1$ efficiency units of labor).

1. Labor demand can be so high that the equilibrium wage is greater than or equal to the wage necessary to motivate even independent workers and ensure full employment. In particular, such an equilibrium requires

$$
L_{D}^{\mathrm{NSM}}\left(w_{t} \mid E_{0}, \mu_{t}^{e}\right)=\left(\mu_{t}^{n} \gamma+\left(1-\mu_{t}^{n}\right)\right)\left(1-E_{0}\right)
$$

for some wage $w_{t} \geq \underline{w}_{i}$ (where $L_{D}^{\mathrm{NSM}}\left(w_{t} \mid E_{0}, \mu_{t}^{e}\right)$ is given by (8)). In this case, the probability that an independent worker will be hired at time $t$ is $q_{t}^{i}=1$.

2. Labor demand is sufficiently low that none of the independent workers will be employed, i.e., $q_{t}^{i}=0$. In particular, when the fraction of independent workers among total workers at time $t$ is $\mu_{t}^{n}$, the condition for such an equilibrium to exist is:

$$
L_{D}^{\mathrm{NSM}}\left(w_{t}=\underline{w}_{i} \mid E_{0}, \mu_{t}^{e}\right) \equiv E_{0}\left(\frac{\varkappa}{\underline{w}_{i}}\right)^{\frac{1}{1-\alpha}}\left[\mu_{t}^{e} A_{i}^{\frac{1}{1-a}}+\left(1-\mu_{t}^{e}\right) A_{o}^{\frac{1}{1-a}}\right]<\left(1-\mu_{t}^{n}\right)\left(1-E_{0}\right) .
$$

Intuitively, this condition implies that at the wage $\underline{w}_{i}$, which is necessary to motivate independent workers, labor demand $L_{D}^{\mathrm{NSM}}\left(w_{t}=\underline{w}_{i} \mid E_{0}, \mu_{t}^{e}\right)$ is less than the total supply of obedient workers, thus ensuring that the equilibrium wage must be strictly less than $\underline{w}_{i}$. In this case, all independent workers will be unemployed, and whether all of the obedient workers will be employed or not depends on whether $L_{D}^{\mathrm{NSM}}\left(w_{t}=\underline{w}_{i} \mid E_{0}, \mu_{t}^{e}\right)$ is less than or greater than $\left(1-\mu_{t}^{n}\right)\left(1-E_{0}\right)$. 
3. Labor demand can be in an intermediate range so that

$$
\left(1-\mu_{t}^{n}\right)\left(1-E_{0}\right) \leq L_{D}^{\mathrm{NSM}}\left(\underline{w}_{i} \mid E_{0}, \mu_{t}^{e}\right)<\left(\mu_{t}^{n} \gamma+\left(1-\mu_{t}^{n}\right)\right)\left(1-E_{0}\right) .
$$

In this case, the equilibrium wage must be $\underline{w}_{i}$, so both obedient and independent workers strictly prefer to be in production work, but there will not be enough demand for all of them. Thus some workers will be forced into unemployment. The rationing rule described above implies that priority in employment will be given to obedient workers, but typically some of the independent workers will also be employed. In particular, we can compute the probability that an independent worker will be hired as

$$
q_{t}^{i}=\frac{L_{D}^{\mathrm{NSM}}\left(\underline{w}_{i} \mid E_{0}, \mu_{t}^{e}\right)-\left(1-\mu_{t}^{n}\right)\left(1-E_{0}\right)}{\mu_{t}^{n} \gamma\left(1-E_{0}\right)} \in[0,1),
$$

which uses the fact that the $\left(1-\mu_{t}^{n}\right)\left(1-E_{0}\right)$ obedient workers will be hired first according to the rationing rule of the competitive equilibrium, and then the remaining labor demand in efficiency units, $L_{D}^{\mathrm{NSM}}\left(\underline{w}_{i} \mid E_{0}, \mu_{t}^{e}\right)-\left(1-\mu_{t}^{n}\right)\left(1-E_{0}\right)$, will be divided among the efficiency units of the independent workers, of which there are $\mu_{t}^{n} \gamma\left(1-E_{0}\right)$ in efficiency units.

I next discuss parenting style decisions of non-entrepreneurs. Imparting independent values (which makes it more likely that their offspring will be independent) has a benefit because of the greater number of efficiency units of labor that independent workers have, but also a cost because independent workers, who require a greater incentive compatible wage, are more likely to be unemployed. Let us now study how this trade-off plays out.

First consider the case in which $q_{t}^{i}=1$. In this case, it is clear that the income of independent workers is greater than that of obedient workers, thus making independent parenting style optimal. Next, if $q_{t}^{i}=0$, then the expected income of an independent individual is zero, and thus obedient parenting style is optimal. In the case where $q_{t}^{i} \in(0,1)$, we know that the equilibrium wage must be $\underline{w}_{i}$, and the expected income of an independent offspring is $q_{t}^{i} \gamma \underline{w}_{i}$, while that of an obedient offspring is $\underline{w}_{i}$ (since obedient workers are employed with probability 1 when $\left.q_{t}^{i}>0\right)$. This leads to a straightforward characterization of the optimal parenting style of non-entrepreneurs:

Claim 2 With no social mobility, non-entrepreneurs impart obedient values to their offspring, i.e., $v_{t-1}^{h^{n}}=v_{o}$, if $q_{t}^{i}<1 / \gamma$; impart independent values, i.e., $v_{t-1}^{h^{n}}=v_{i}$, if $q_{t}^{i}>1 / \gamma$; and are indifferent between parenting styles when $q_{t}^{i}=1 / \gamma{ }^{13}$

\footnotetext{
${ }^{13}$ Note the timing. Parents at time $t-1$ are choosing parenting style taking into account the labor market equilibrium at time $t$. None of the key conclusions depend on this forward-looking parenting style, and myopic (backward-looking) choice of parenting style would lead to similar results.
} 
To make further progress, let us find the combinations of $\mu_{t}^{e}$ and $\mu_{t}^{n}$ such that $q\left(\mu_{t}^{e}, \mu_{t}^{n}\right)=$ $1 / \gamma$. Note that

$$
q\left(\mu_{t}^{e}, \mu_{t}^{n}\right)=\max \left\{\min \left[\frac{E_{0}\left(\frac{\varkappa}{\underline{w}_{i}}\right)^{\frac{1}{1-\alpha}}\left[\mu_{t}^{e} A_{i}^{\frac{1}{1-a}}+\left(1-\mu_{t}^{e}\right) A_{o}^{\frac{1}{1-a}}\right]-\left(1-\mu_{t}^{n}\right)\left(1-E_{0}\right)}{\mu_{t}^{n} \gamma\left(1-E_{0}\right)} ; 1\right] ; 0\right\},
$$

where the maximum and minimum make sure that $q\left(\mu_{t}^{e}, \mu_{t}^{n}\right)$ remains between 0 and 1 . Setting this expression equal to $1 / \gamma$, we find that when $q\left(\mu_{t}^{e}, \mu_{t}^{n}\right)=1 / \gamma$ (which is of course less than 1), we have

$$
\frac{E_{0}\left(\frac{\varkappa}{\underline{w}_{i}}\right)^{\frac{1}{1-\alpha}}\left[\mu_{t}^{e} A_{i}^{\frac{1}{1-a}}+\left(1-\mu_{t}^{e}\right) A_{o}^{\frac{1}{1-a}}\right]-\left(1-\mu_{t}^{n}\right)\left(1-E_{0}\right)}{\mu_{t}^{n} \gamma\left(1-E_{0}\right)}=\frac{1}{\gamma},
$$

or

$$
E_{0}\left(\frac{\varkappa}{\underline{w}_{i}}\right)^{\frac{1}{1-\alpha}}\left[\mu_{t}^{e} A_{i}^{\frac{1}{1-a}}+\left(1-\mu_{t}^{e}\right) A_{o}^{\frac{1}{1-a}}\right]=\left(1-E_{0}\right)
$$

which does not depend on $\mu_{t}^{n}$. This implies that whenever $E_{0}\left(\frac{\varkappa}{\underline{w}_{i}}\right)^{\frac{1}{1-\alpha}}\left[\mu_{t}^{e} A_{i}^{\frac{1}{1-a}}+\left(1-\mu_{t}^{e}\right) A_{o}^{\frac{1}{1-a}}\right]<1-E_{0}$, we have $q_{t}^{i}<1 / \gamma$, and whenever $E_{0}\left(\frac{\varkappa}{\underline{w}_{i}}\right)^{\frac{1}{1-\alpha}}\left[\mu_{t}^{e} A_{i}^{\frac{1}{1-a}}+\left(1-\mu_{t}^{e}\right) A_{o}^{\frac{1}{1-a}}\right]>1-E_{0}, q_{t}^{i}>1 / \gamma$. Defining $\tilde{\mu}^{e}$ such that ${ }^{14}$

$$
E_{0}\left(\frac{\varkappa}{\underline{w}_{i}}\right)^{\frac{1}{1-\alpha}}\left[\tilde{\mu}^{e} A_{i}^{\frac{1}{1-a}}+\left(1-\tilde{\mu}^{e}\right) A_{o}^{\frac{1}{1-a}}\right]=1-E_{0}
$$

the following stronger version of Claim 2 is obtained.

Claim 3 If $\mu_{t}^{e}>\tilde{\mu}^{e}$, then all non-entrepreneurs prefer independent parenting style, i.e., $v_{t-1}^{h^{n}}=$ $v_{o}$; and if $\mu_{t}^{e}<\tilde{\mu}^{e}$, then non-entrepreneurs prefer obedient parenting style, i.e., $v_{t-1}^{h^{n}}=v_{i}$.

Conveniently, these relationships are independent from the fraction of independent types among non-entrepreneurs, $\mu_{t}^{n}$, and depend only on the fraction of independent types among entrepreneurs, $\mu_{t}^{e}$. This enables a simple graphical analysis of dynamics in the $\left(\mu_{t}^{n}, \mu_{t}^{e}\right)$ space, which will be depicted in Figure 4. Before presenting this analysis, it is useful to determine the dynamics of the fraction of non-entrepreneurs that are independent. This fraction can be written as

$$
\mu_{t}^{n}=\mathbf{I}\left(\mu_{t}^{e}>\tilde{\mu}^{e}\right)\left(\pi_{i i} \mu_{t-1}^{n}+\pi_{o i}\left(1-\mu_{t-1}^{n}\right)\right)+\mathbf{I}\left(\mu_{t}^{e}<\tilde{\mu}^{e}\right)\left(\pi_{i o} \mu_{t-1}^{n}+\pi_{o o}\left(1-\mu_{t-1}^{n}\right)\right),
$$

\footnotetext{
${ }^{14}$ Clearly $\tilde{\mu}^{e}$ is a function of $E_{0}$, but I suppress this dependence to simplify notation.
} 
where $\mathbf{I}\left(\mu_{t}^{e}>\tilde{\mu}^{e}\right)$ is the indicator function which determines whether non-entrepreneur parents choose independent or obedient values for their parenting style, and for simplicity, I have not explicitly written the intermediate case where $\mu_{t}^{e}=\tilde{\mu}^{e}$, where the value of $\mu_{t}^{n}$ is not uniquely determined since parents are indifferent between obedient and independent parenting styles. It is clear from 12 that depending on whether $\mu_{t}^{e}$ is greater than or less than $\tilde{\mu}^{e}, \mu_{t}^{n}$ will tend to a steady-state value of either

$$
\bar{\mu}_{i}^{n}=\frac{\pi_{o i}}{1-\pi_{i i}+\pi_{o i}} \text { or } \bar{\mu}_{o}^{n}=\frac{\pi_{o o}}{1-\pi_{i o}+\pi_{o o}},
$$

where, of course, $\bar{\mu}_{i}^{n}$ is the same as $\bar{\mu}^{e}$ in $(7)$, since in both cases all individuals are choosing independent parenting. Note also that $\bar{\mu}_{i}^{n}$ in $(13)$ is the asymptotic limit when $\mu_{t}^{e}>\tilde{\mu}^{e}$, while $\bar{\mu}_{o}^{n}$ is the limit when $\mu_{t}^{e}<\tilde{\mu}^{e}$.

Now returning to Figure 4, from Claim 1, entrepreneurs always choose to impart independent values to their offspring, so the dynamics of $\mu_{t}^{e}$ monotonically converge to $\bar{\mu}^{e}$, which is marked as a horizontal line in the figure. Another horizontal line is placed at $\tilde{\mu}^{e}$. We then separately treat two cases where $\bar{\mu}^{e}<\tilde{\mu}^{e}$ and $\bar{\mu}^{e}>\tilde{\mu}^{e}$. These are respectively equivalent to $E_{0}\left(\frac{\varkappa}{\underline{w}_{i}}\right)^{\frac{1}{1-\alpha}}\left[\bar{\mu}^{e} A_{i}^{\frac{1}{1-a}}+\left(1-\bar{\mu}^{e}\right) A_{o}^{\frac{1}{1-a}}\right]$ being greater than or less than $1-E_{0}$.
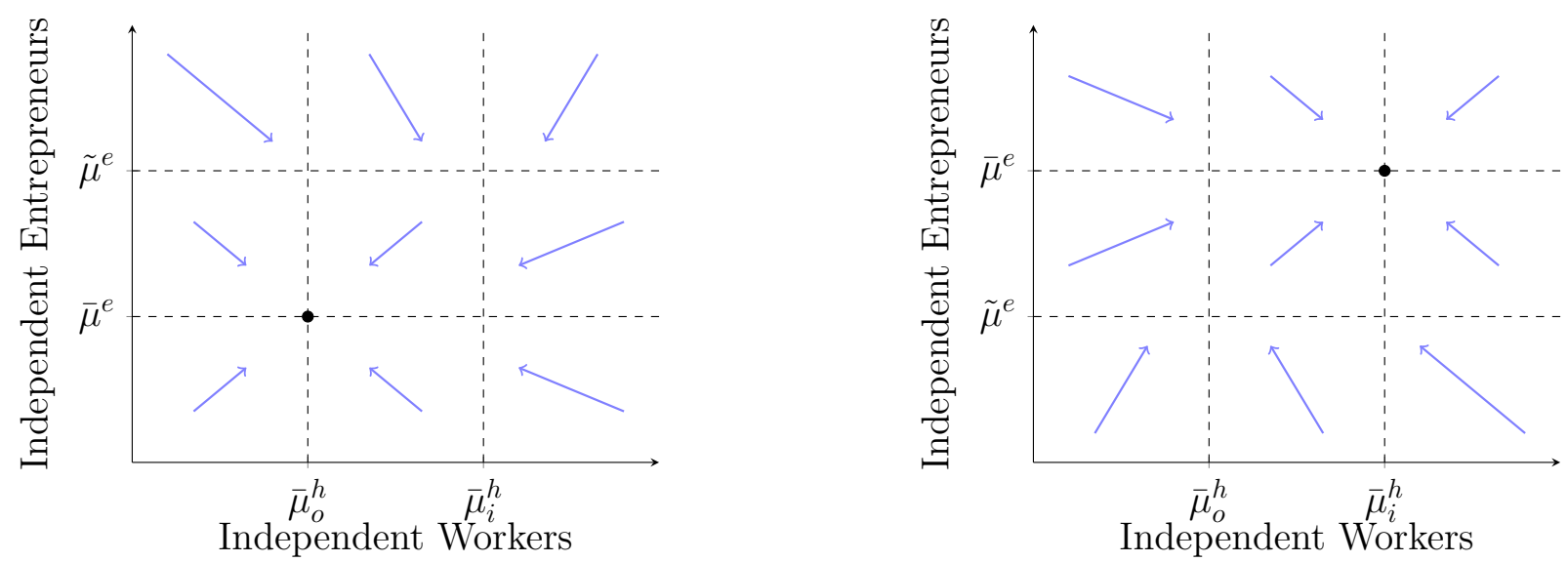

Figure 4: Dynamics of Fraction of Independent Entrepreneurs and Workers

The left panel of Figure 4 corresponds to the case where $\bar{\mu}^{e}<\tilde{\mu}^{e}$, while the right panel is for $\bar{\mu}^{e}>\tilde{\mu}^{e}$. In each case, the arrows show the direction of change of $\mu_{t}^{e}$ and $\mu_{t}^{n}$. In particular, when $\mu_{t}^{e}<\bar{\mu}^{e}, \mu_{t}^{e}$ will increase and vice versa. Also, when $\mu_{t}^{e}>\tilde{\mu}^{e}, \mu_{t}^{n}$ will increase (until it reaches $\bar{\mu}_{o}^{n}$ ) and when $\mu_{t}^{e}<\tilde{\mu}^{e}, \mu_{t}^{n}$ will decrease (until it reaches $\bar{\mu}_{i}^{n}$ ). The two panels 
show that there is a unique steady-state equilibrium given by the intersection of $\bar{\mu}^{e}$ with the vertical portions of the line for the optimal parenting decision of non-entrepreneurs. Note that throughout the arrows are also uniquely determined, indicating that the dynamic equilibrium is unique as well. This is despite the fact that payoffs to a parent depend on the parenting style of other parents. These results follow from Claims 1 and 3 , which make parenting style decisions functions of $\mu_{t}^{e}$ (and not a function of $\mu_{t}^{n}$ ). Finally, once the distribution of types is determined, it is straightforward to characterize the equilibrium wage (in or out of steady state) from the above characterization.

This diagrammatic analysis establishes:

Proposition 1 Suppose that Assumptions 1 and 2 hold and there is no social mobility. Then starting with any initial condition $\left(\mu_{0}^{n}, \mu_{0}^{e}\right)$, there exists a unique dynamic equilibrium. In this equilibrium, whenever $\tilde{\mu}^{e}<\mu_{t}^{e}$ all parents choose independent parenting style, i.e., $v_{t}^{h}=v_{i}$ for all $h$, and whenever $\tilde{\mu}^{e}>\mu_{t}^{e}$ entrepreneurs choose independent parenting style, i.e., $v_{t}^{h^{e}}=v_{i}$, while non-entrepreneurs choose obedient parenting style, i.e., $v_{t}^{h^{n}}=v_{o}$.

Moreover, if $\tilde{\mu}^{e}<\bar{\mu}^{e}$, then the unique equilibrium path converges to a steady state in which all parents choose independent parenting style, i.e., $v^{h}=v_{i}$ for all $h$, and the equilibrium wage rate is given by $w^{e}=w \geq \underline{w}_{i}$ where

$$
E_{0}\left(\frac{\varkappa}{w}\right)^{\frac{1}{1-\alpha}}\left[\bar{\mu}^{e} A_{i}^{\frac{1}{1-a}}+\left(1-\bar{\mu}^{e}\right) A_{o}^{\frac{1}{1-a}}\right]=\left(1-E_{0}\right)\left(1+(\gamma-1) \bar{\mu}^{e}\right) .
$$

If $\tilde{\mu}^{e}>\bar{\mu}^{e}$, then the unique equilibrium path converges to a steady state in which all entrepreneurs choose independent parenting style, i.e., $v^{h^{e}}=v_{i}$, and non-entrepreneurs choose obedient parenting style, i.e., $v^{h^{n}}=v_{o}$. If

$$
E_{0}\left(\frac{\varkappa}{\underline{w}_{i}}\right)^{\frac{1}{1-\alpha}}\left[\bar{\mu}^{e} A_{i}^{\frac{1}{1-a}}+\left(1-\bar{\mu}^{e}\right) A_{o}^{\frac{1}{1-a}}\right]<\left(1-E_{0}\right)\left(1-\bar{\mu}^{e}\right),
$$

then the equilibrium wage rate is $w^{e} \in\left[\underline{w}_{o}, \underline{w}_{i}\right)$, and if this inequality is reversed, then the equilibrium wage rate is $\underline{w}_{i}$.

Proposition 1 characterizes a situation in which entry barriers keep a group of dynasties as the sole entrepreneurs, while the rest of the population is either employed as workers or unemployed.

The key result of this proposition, and in some sense the main result of the paper as anticipated in the Introduction, is within-society cultural heterogeneity: entrepreneurial dynasties 
adopt a different parenting style than the rest of society. Recognizing their privileged position (in particular, the fact that their children will become entrepreneurs), they impart values of independence to them, which will be useful in entrepreneurship. In contrast, non-entrepreneurial dynasties recognize their disadvantaged (social and economic) position and understand that their offspring will be better off having obedient values so that they adapt better to production work, and as a result impart values emphasizing obedience.

However, Proposition 1 also emphasizes that this divergence is not because of imperfect empathy making parents choose investments to make their offspring similar to themselves (as in Bisin and Verdier, 2000, or Tabellini, 2009), but depends on the nature of the economic equilibrium. When $\tilde{\mu}^{e}<\mu_{t}^{e}$, the productivity advantage of independent workers is more than offset by their lower likelihood of finding employment, and all non-entrepreneur parents choose an obedient parenting style. In contrast, when $\tilde{\mu}^{e}>\mu_{t}^{e}$, the number of entrepreneurs is sufficiently high and non-entrepreneurial families also prefer independent parenting styles, because this makes their offspring more productive.

Proposition 1 highlights that this divergence in values imparted by families depends on the nature of the economic equilibrium. It does not arise when labor demand is sufficiently high because in this case there is no rationing and thus no advantage for obedient workers. It also does not arise when there are no entry barriers because then the offspring of non-entrepreneurs could become entrepreneurs, encouraging independent parenting by all parents (as we will see in the next subsection). However, when entry barriers close off this vocation to the children of non-entrepreneurs and labor demand is low, the optimal parenting style of these agents changes and places greater emphasis on obedience.

\subsection{Equilibrium with Social Mobility}

I next study this economy when entry barriers, $B_{t}$, are not prohibitive and there is exit from entrepreneurship (and in the process also derive conditions for the "no social mobility" equilibrium studied in the previous subsection). To simplify the exposition and some of the details of the analysis, I will focus on steady-state equilibria and then comment on local dynamics. Given this steady-state focus, I parameterize $B_{t}=b_{t} w_{t}$ and take $b_{t}=b{ }^{15}$ I will also drop time subscripts whenever this will cause no confusion.

\footnotetext{
${ }^{15}$ This assumes that entry costs are proportional to the wage, which could be because they involve labor (though I am not modeling this and do not include it as part of the labor market clearing condition) or because bribes or other inefficient transfers that need to be paid increase with the wage.
} 
Let us first consider the problem of an independent worker contemplating entry into entrepreneurship. If she remains a non-entrepreneur, her expected income is

$$
q \gamma w
$$

and if she enters into entrepreneurship, her income is given by equation (5) above minus the cost of entry, or

$$
w^{-\frac{\alpha}{1-\alpha}} A_{i}^{\frac{1}{1-\alpha}}-b w
$$

The comparison of these two expressions will depend on whether independent workers are rationed or not. Let us first take the worst case scenario for independent workers, in which labor demand is so low that $q=0$ and $w$ takes the minimum (incentive-compatible) value, $\underline{w}_{o}$. Even then, if the entry cost is greater than the threshold

$$
\bar{b} \equiv\left(\frac{A_{i}}{\underline{w}_{o}}\right)^{\frac{1}{1-\alpha}},
$$

there will never be any entry into entrepreneurship by non-entrepreneurs. Hence, we trivially have (where the weak inequality is without loss of generality):

Claim 4 If $b \geq \bar{b}$, then there is never any entry of non-entrepreneurs into entrepreneurship .16

To study the labor market equilibrium and parenting styles when $b<\bar{b}$, I next introduce a number of additional thresholds. First, let us suppose that there is full employment of independent workers. Then in (14), we have $q=1$, and the lowest (incentive-compatible) wage is $w=\underline{w}_{i}$. At this lowest feasible wage, the comparison for a non-entrepreneur when it comes to deciding whether or not to enter into entrepreneurship boils down to:

$$
\gamma \underline{w}_{i} \leq \underline{w}_{i}^{-\frac{\alpha}{1-\alpha}} A_{i}^{\frac{1}{1-\alpha}}-b \underline{w}_{i} .
$$

Setting the two sides of this inequality equal to each other, we obtain another threshold for entry barriers:

$$
b^{*} \equiv\left(\frac{A_{i}}{\underline{w}_{i}}\right)^{\frac{1}{1-\alpha}}-\gamma .
$$

When $b<b^{*}$ and $w=\underline{w}_{i}$, all independent non-entrepreneurs would prefer to enter entrepreneurship.

\footnotetext{
${ }^{16}$ This implies that for any $\varepsilon>0$, the number of entrepreneurs will converge to zero in the very distant future. But in what follows, I focus on the limit case where $\varepsilon=0$, using $\varepsilon \rightarrow 0$ as a selection device, as pointed out above.
} 
The question then becomes whether independent types will enter into entrepreneurship once the wage adjusts. To answer this question, I first define the wage $w^{*}(b)$ such that, when the entry barrier is equal to $b$ and there is full employment, independent non-entrepreneurs are indifferent between entering into entrepreneurship and working:

$$
\gamma w^{*}(b)=w^{*}(b)^{-\frac{\alpha}{1-\alpha}} A_{i}^{\frac{1}{1-\alpha}}-b w^{*}(b) .
$$

In particular, the left-hand side is the income of independent types under full employment, while the right-hand side is the difference between their profits at this wage minus the cost of entry. Rearranging terms in this equation yields: ${ }^{17}$

$$
w^{*}(b) \equiv A_{i}(b+\gamma)^{-(1-\alpha)} .
$$

With this expression at hand, I now introduce two more assumptions.

\section{Assumption 3}

$$
\bar{\mu}^{e} \varkappa^{\frac{1}{1-\alpha}} \gamma>1-\bar{\mu}^{e}
$$

To interpret this inequality, first suppose that all parents choose to impart values of independence to their offspring. In this case, if all independent types (whose population share in steady state is $\left.\bar{\mu}^{e}\right)$ were to enter entrepreneurship, then labor demand at the wage $w^{*}(0)$, as defined by (16)), would be given by the left-hand side of this expression. Labor supply, on the other hand, would be made up of at most $1-\bar{\mu}^{e}$ obedient workers, each supplying one unit of labor, as shown on the right-hand side. Because labor demand is decreasing in the wage and $w^{*}(b)$ is decreasing in $b$, labor demand will be even higher at all entry barriers $b \in\left(0, b^{*}\right)$, and thus Assumption 3 is sufficient to ensure that there would also be excess demand for labor at all entry costs $b \leq b^{*}$ if all independent types entered entrepreneurship.

Moreover, in this case, all agents will indeed choose independent parenting, because we are looking at an equilibrium in which there is sufficient entry into entrepreneurship and thus full employment. Given full employment, independent parenting is a dominant decision even for non-entrepreneurs. More broadly, parenting decisions will be straightforward in this environment with social mobility. Because, as we will see, in steady state there will be either no entry into entrepreneurship or, when there is entry, non-entrepreneurs will be indifferent between production work and entrepreneurship. In both cases, therefore, the parenting choice

\footnotetext{
${ }^{17}$ It can be verified that $w^{*}(b)<\bar{w} \equiv A_{i} \gamma^{-(1-\alpha)}$, which is the maximal wage consistent with any agent considering entry into entrepreneurship (even with zero entry cost), and also $w^{*}(b)>\underline{w}_{i}$ for all $b<b^{*}$.
} 
of non-entrepreneurs will come down to whether an independent offspring can find employment with sufficiently high probability (if the answer is yes, independent parenting is preferred, and if it is no, obedient parenting is preferred).

Assumption 3 implies that the (steady-state) equilibrium wage can never be below $w^{*}(b)$ when entry barriers are given by $b$. If it were below it, then all independent types, including those who are currently non-entrepreneurs, would prefer entrepreneurship. This is inconsistent with an equilibrium as I argue next. First, we cannot have $w \leq \underline{w}_{i}$ and $w<w^{*}(b)$, because in this case obedient entrepreneurs would prefer to remain in entrepreneurship as well, which is inconsistent with equilibrium, since labor demand would exceed even the left-hand side of Assumption 3. Therefore, we must have $w>\underline{w}_{i}$, but in this case, all parents, including nonentrepreneurs, choose independent values, and from Assumption 3, labor market clearing is again violated. Thus we conclude that $w \geq w^{*}(b)$.

Can $w$ be strictly above $w^{*}(b)$ ? This would be the case if the demand for labor were sufficiently high, even without any of the independent non-entrepreneurs entering into entrepreneurship (because when the wage is above this threshold, there is zero entry from this group). The feasibility of this depends on whether obedient entrepreneurs (obedient offspring of entrepreneur parents) stay in entrepreneurship. They will prefer to do so, when the entry barrier is sufficiently high, pushing wages down to very low levels. Let us define the threshold level of entry barriers for this as $b^{* *}$ such that $A_{o}=w^{*}\left(b^{* *}\right)$, or

$$
b^{* *} \equiv\left(\frac{A_{i}}{A_{o}}\right)^{\frac{1}{1-\alpha}}-\gamma<b^{*} .
$$

This expression follows by substituting for $w^{*}\left(b^{* *}\right)$ from 16 . By definition, when $b \geq b^{* *}$ obedient types prefer to remain in entrepreneurship. Conversely, when $b<b^{* *}$, they prefer to exit entrepreneurship. But this is inconsistent with $w>w^{*}(b)$. To see why, note that $w>w^{*}(b)$ implies that $w>w^{*}\left(b^{* *}\right)$, and with the same argument as in the previous paragraph, $w>\underline{w}_{i}$. This would imply, once again, that all independent non-entrepreneurs prefer production work and do not enter entrepreneurship. On the other hand, because $b<b^{* *}$ obedient types exit entrepreneurship, which is inconsistent with a steady state ${ }^{18}$

Therefore, the wage must be exactly equal to $w^{*}(b)$, which will support full employment

\footnotetext{
${ }^{18}$ This argument again exploits the fact that for any $\varepsilon>0$, we need some entry into entrepreneurship. When the wage were above $w^{*}(b)$, the argument in the text establishes that this entry would not be forthcoming and the number of entrepreneurs would gradually decline towards zero. Hence, for any $\varepsilon>0$, the equilibrium must have some exit and thus the wage must be at $w^{*}(b)$. As before, I then take the limit $\varepsilon \rightarrow 0$ and focus on the limit equilibrium in which the wage is equal to $w^{*}(b)$.
} 
(because once again $w>\underline{w}_{i}$ ) and thus independent parenting by all agents. Finally, since obedient types exit entrepreneurship, all entrepreneurs are independent types, which gives us:

Claim 5 Suppose Assumptions 1-4 hold. Then, when $b<b^{* *}$, the steady-state equilibrium involves full employment, an equilibrium wage of $w^{*}(b)$, independent parenting style by all types and exit of all obedient types from entrepreneurship. The measure of entrepreneurs is

$$
E_{\text {exit }}^{S}(b)\left(\frac{\varkappa}{w^{*}(b)}\right)^{\frac{1}{1-\alpha}} A_{i}^{\frac{1}{1-a}}=1-\bar{\mu}^{e}+\left(\bar{\mu}^{e}-E_{\text {exit }}^{S}(b) \gamma\right) .
$$

The expression for the steady-state employment level, $E_{\text {exit }}^{S}(b)$, follows by noting that (i) all entrepreneurs are independent, which explains the left-hand side; (ii) all agents are choosing independent parenting, which implies that the population fraction of independent types is $\bar{\mu}^{e}$; and (iii) as a result, workers consist of $1-\bar{\mu}^{e}$ obedient types and the independent types who are not in entrepreneurship, $\bar{\mu}^{e}-E_{\text {exit }}^{S}(b)$, who supply $\gamma$ units of labor.

Let us next consider the case where $b \in\left(b^{* *}, b^{*}\right)$. With the same argument, the wage can never fall below $w^{*}(b)$. Can it exceed $w^{*}(b)$ in this range? I now impose one more assumption, which will ensure that the answer is again no. In particular, I impose that the productivity gap between independent and obedient entrepreneurs is large enough that at the wage $w^{*}\left(b^{* *}\right)$, when independent non-entrepreneurs are indifferent between entrepreneurship and production work, obedient types strictly prefer not to enter entrepreneurship:

\section{Assumption 4}

$$
\frac{A_{i}}{A_{o}}>\left(\frac{b^{* *}+\gamma}{b^{* *}+1}\right)^{1-\alpha}
$$

Since the right-hand side of this expression is decreasing in $b$, it also ensures that only independent types would consider entering entrepreneurship for all $b \geq b^{* *}$. Moreover, by definition, in this range, obedient entrepreneurs do not exit entrepreneurship. If we had $w>$ $w^{*}(b)$, there would be no entry into entrepreneurship at all, which is not consistent with a steady state.

This argument establishes:

Claim 6 Suppose Assumptions 1-4 hold. Then, when $b \in\left(b^{* *}, b^{*}\right)$, the steady-state equilibrium involves full employment, an equilibrium wage of $w^{*}(b)$, independent parenting style by all types, no exit from entrepreneurship by obedient types and no new entry. The measure of entrepreneurs is $E^{S}(b)$, given by

$$
E^{S}(b)\left(\frac{\varkappa}{w^{*}(b)}\right)^{\frac{1}{1-\alpha}}\left[\bar{\mu}^{e} A_{i}^{\frac{1}{1-a}}+\left(1-\bar{\mu}^{e}\right) A_{o}^{\frac{1}{1-a}}\right]=\left(1-E^{S}(b)\right)\left(1-\bar{\mu}^{e}+\gamma \bar{\mu}^{e}\right) .
$$


The steady-state number of entrepreneurs $E^{S}(b)$ differs from $(18)$, because now there is no exit of obedient entrepreneurs, and as a result, the steady-state fraction of independent types among both entrepreneurs and non-entrepreneurs is $\bar{\mu}^{e}$ (since both entrepreneurs and non-entrepreneurs are choosing independent parenting style and there is no exit or entry).

Finally, let us return to entry barriers in the range $b \in\left(b^{*}, \bar{b}\right)$. Since $b>b^{*}$, we will no longer have full employment. If we had full employment even at the lowest possible wage consistent with it, $w=\underline{w}_{i}$, then independent non-entrepreneurs would prefer not to enter entrepreneurship (and from Assumption 4, obedient types would also prefer not to enter), and this would be inconsistent with labor market clearing in the long run. As a result, in any steady state in this range we must have $w \leq \underline{w}_{i}$ and some unemployment, and this level of unemployment must be such that independent non-entrepreneurs are just indifferent between entering and not entering entrepreneurship (this again follows from the same argument; if they all preferred entrepreneurship or if they all preferred non-entrepreneurship, this would not be consistent with long-run labor market equilibrium). Thus, to characterize steady-state equilibria in this range, all we need to do is to find the value of rationing probability $q$ such that independent non-entrepreneurs are indifferent between production work and entrepreneurship.

With this purpose, let us first define a new threshold, $\tilde{b}(q)$ such that for entry barriers above this threshold, non-entrepreneurs do not want to enter into entrepreneurship when the wage is $\underline{w}_{i}$ and the probability of an independent worker getting a job is $q$. In particular, entrepreneurship has a net income of $\underline{w}_{i}^{-\frac{\alpha}{1-\alpha}} A_{i}^{\frac{1}{1-\alpha}}-b \underline{w}_{i}$, and setting this equal to $q \gamma \underline{w}_{i}$, we obtain

$$
\tilde{b}(q) \equiv\left(\frac{A_{i}}{\underline{w}_{i}}\right)^{\frac{1}{1-\alpha}}-q \gamma<\bar{b} .
$$

First consider $b \in\left(b^{*}, \tilde{b}(1 / \gamma)\right]$. In this range, by construction, there will be no entry of entrepreneurs provided that the probability of an independent type getting a production job is $q=\tilde{b}^{-1}(b)$ and the wage is $\underline{w}_{i}$ (since by definition $b>b^{* *}$ ). Because the probability of an independent worker getting a job is greater than $1 / \gamma$, non-entrepreneurs will also choose independent parenting. Moreover, the wage cannot fall below $\underline{w}_{i}$, because if it did, then it would mean that no independent type would be able to get production work, and in this range of parameters, all independent types would then prefer to enter entrepreneurship. Because there is no exit in this range and both entrepreneurs and non-entrepreneurs are choosing independent parenting, the equilibrium measure of entrepreneurs will then have to satisfy:

$$
\tilde{E}^{S}(q)\left(\frac{\varkappa}{\underline{w}_{i}}\right)^{\frac{1}{1-\alpha}}\left[\bar{\mu}^{e} A_{i}^{\frac{1}{1-a}}+\left(1-\bar{\mu}^{e}\right) A_{o}^{\frac{1}{1-a}}\right]=\left(1-\tilde{E}^{S}(q)\right)\left(1-\bar{\mu}^{e}+q \gamma \bar{\mu}^{e}\right),
$$


which has a reasoning analogous to (19), except that on the right-hand side, there is explicit allowance for the fact that only a fraction $q$ of the independent types will be employed.

When $b \in(\tilde{b}(1 / \gamma),(\tilde{b}(0)))$, the argument is similar to the previous case, except that now non-entrepreneurs choose obedient parenting. With the same argument, the wage cannot fall below $\underline{w}_{i}$. Hence, there must be just enough labor demand in steady states to ensure labor market clearing, which is now given by:

$$
\hat{E}^{S}(q)\left(\frac{\varkappa}{\underline{w}_{i}}\right)^{\frac{1}{1-\alpha}}\left[\bar{\mu}^{e} A_{i}^{\frac{1}{1-a}}+\left(1-\bar{\mu}^{e}\right) A_{o}^{\frac{1}{1-a}}\right]=\left(1-\hat{E}^{S}(q)\right)\left(1-\bar{\mu}_{o}^{n}+q \gamma \bar{\mu}_{o}^{n}\right),
$$

where the main difference from $(21)$ is that on the right-hand side we have $\bar{\mu}_{o}^{n}$ as given in (13), since non-entrepreneurs are now choosing obedient parenting.

Finally, when $b \in(\tilde{b}(0), \bar{b})$, independent workers will be completely rationed out of production jobs. As a result, the wage can now be less than $\underline{w}_{i}$. Depending on the exact level of the wage, it may be profitable for these independent non-entrepreneurs to enter entrepreneurship (since profits in entrepreneurship depend on wages). But with similar reasoning to the one we have used so far, if they all decided to enter entrepreneurship, this would be inconsistent with equilibrium. Therefore, the wage has to be such that non-entrepreneurs prefer not to enter. Let $\bar{b}(w)$ be defined as

$$
\bar{b}(w) \equiv\left(\frac{A_{i}}{w}\right)^{\frac{1}{1-\alpha}} \text { for } w \in\left(\underline{w}_{o}, \underline{w}_{i}\right] .
$$

Then, in this case, the steady-state equilibrium must have a wage $w=\bar{b}^{-1}(b)$, and independent non-entrepreneurs are indifferent between entering and not entering entrepreneurship 19 With the same reasoning as in the preceding, non-entrepreneurs will choose obedient parenting, and thus market clearing entails:

$$
\bar{E}^{S}(w)\left(\frac{\varkappa}{w}\right)^{\frac{1}{1-\alpha}}\left[\bar{\mu}^{e} A_{i}^{\frac{1}{1-a}}+\left(1-\bar{\mu}^{e}\right) A_{o}^{\frac{1}{1-a}}\right]=\left(1-\bar{E}^{S}(w)\right)\left(1-\bar{\mu}_{o}^{n}\right),
$$

Summarizing these results, we have:

Claim 7 Suppose Assumptions 1-4 hold. Let $\tilde{b}(q)$ be as given in (20) and $\bar{b}(w)$ be as given in (23).

1. When $b \in\left[b^{*}, \tilde{b}(1 / \gamma)\right]$, in the unique steady-state equilibrium, there is no entry into or exit from entrepreneurship, the wage is $\underline{w}_{i}$, a fraction $q=\tilde{b}^{-1}(b)$ of independent

\footnotetext{
${ }^{19}$ The reason why the wage cannot be higher is once again that if it were, then for any $\varepsilon>0$, we could not have a steady-state equilibrium.
} 
workers find a job, all agents choose independent parenting, and the equilibrium measure of entrepreneurs is given by $\tilde{E}^{S}(q)$ as in (21).

2. When $b \in[\tilde{b}(1 / \gamma), \tilde{b}(0))$, in the unique steady-state equilibrium, there is no entry into or exit from entrepreneurship, the wage is $\underline{w}_{i}$, a fraction $q=\tilde{b}^{-1}(b)$ of independent workers find a job, entrepreneurs choose independent parenting and non-entrepreneurs choose obedient parenting, and the equilibrium measure of entrepreneurs is given by $\hat{E}^{S}(q)$ as in (22).

3. When $b \in(\tilde{b}(0), \bar{b}]$, in the unique steady-state equilibrium, there is no entry into or exit from entrepreneurship, the wage is $w=\bar{b}^{-1}(b)$, independent workers are fully rationed out of production jobs, entrepreneurs choose independent parenting and non-entrepreneurs choose obedient parenting, and the equilibrium measure of entrepreneurs is given by $\bar{E}(w)$ as in 24).

We can now put the previous four claims together to obtain the main result of this subsection:

Proposition 2 Suppose that Assumptions 1-4 hold. Let $b^{*}, b^{* *}, \tilde{b}(q)$ and $\bar{b}(w)$ be given by (15), (17), 20) and (23).

1. If $b>\bar{b}$, there is no social mobility and steady-state equilibria are given by Proposition 1 .

2. If $b \in\left[b^{*}, \bar{b}\right]$, there is no social mobility and Claim 7 characterizes steady-state equilibria.

3. If $b^{* *}<b<b^{*}$, there is a unique steady-state equilibrium where there is full employment, all parents choose independent parenting style, i.e., $v_{t}^{h}=v_{i}$ for all $h$ and $t$, there is no exit of entrepreneurs, the total measure of entrepreneurs is $E^{S}(b)$ given by (19), the fraction of independent types among entrepreneurs is $\bar{\mu}^{e}$ given by (10), and the equilibrium wage is $w^{*}(b)$ given by $(16)$.

4. If $b<b^{* *}$, there is a unique steady-state equilibrium where there is full employment, $\mu_{t}^{e}=1$ (i.e., entry of independent entrepreneurs and exit of obedient entrepreneurs), the total measure of entrepreneurs is $E_{\text {exit }}^{S}(b)$ given by (18), all parents choose to impart independent values, i.e., $v_{t}^{h}=v_{i}$ for all $h$ and $t$ ), and the steady-state equilibrium wage is $w^{*}(b)$ given by $(16)$. 
Most importantly, part 4 of Proposition 2 characterizes an equilibrium in which, with nonprohibitive entry barriers, there is some entry of independent types into entrepreneurship. As we will see in the next section, the most relevant parts are 1 and 4, because, when entry barriers are endogenized, we will be in one of these two cases. Nevertheless, the intermediate cases also highlight some of the economic forces in the model.

The most important economic lessons are that depending on the extent of entry barriers, the cultural configurations that arise can be very different than those in Proposition 1. Specifically, we saw in Proposition 1 that with prohibitive entry barriers and low initial stock of entrepreneurs, non-elite/non-entrepreneur parents choose obedient values for their children, generating an endogenous source of income inequality (lower incomes for obedient children of non-elite parents) and social hierarchy (children of entrepreneurs more likely to be entrepreneurs/bosses). In contrast, a very different social equilibrium emerges when entry barriers are low as in part 4 of this proposition. In this case, parents expect better economic opportunities for their children and choose independent parenting. In contrast to Proposition 1. the endogenous source of inequality between children of elite and non-elite parents is significantly lessened - since both types of parents choose to impart independent values, which makes their offspring more productive and even the offspring of non-entrepreneurs can enter entrepreneurship, increasing their incomes both directly and also indirectly because of higher equilibrium wages. This result highlights the fluidity of cultural configurations in the face of different incentives created by the labor market equilibrium.

Remark 1 Proposition 2 focuses on steady-state equilibria. Although equilibrium dynamics are more involved, the following argument establishes that starting from $E_{0}$ in the neighborhood of the relevant steady state $E^{S}$, the dynamic equilibrium converges to the steady states characterized in this proposition. Take, for example, part 4 of the proposition. Suppose that $E_{0}$ is close to but less than $E^{S}$. Then, labor demand will be sufficiently high that non-entrepreneurs still prefer independent parenting, and because $b<b^{* *}$, there will be entry of independent types into entrepreneurship, gradually increasing the number of entrepreneurs towards the steady state. If, instead, $E_{0}$ had been much lower than $E^{S}$, then non-entrepreneurs might have preferred obedient parenting because labor demand is low. Nevertheless, in this case there would again be convergence to the steady-state equilibrium. In other cases covered in the proposition, local dynamics converge to the steady state as well, but global dynamics may be more complicated because away from the steady state, parenting or entrepreneurial decisions may not be uniquely defined. 
Remark 2 Although the steady-state equilibrium characterized so far is unique, there are forces that might have led to multiple equilibria or multiple steady states this model. In particular, when the equilibrium wage is low, non-entrepreneurs may be discouraged from imparting independent values because they expect their offspring to be rationed out of employment in the labor market, and this might reduce productive entrepreneurship, keeping wages low. However, under the baseline assumptions adopted so far, this effect is always dominated, because when the equilibrium wage is low, entrepreneurship becomes more profitable, and non-entrepreneurs may choose independent parenting, expecting that their children will choose to become entrepreneurs. If we generalize the model by making entrepreneurship opportunities stochastic, then multiplicity can arise. For example, suppose that a non-entrepreneur has an opportunity to enter into partnership with with probability $\lambda \leq 1$ (so that the baseline model is the special case with $\lambda=1$ ). In this case, low wages will discourage independent parenting for non-entrepreneurs, even when they would like their offspring to enter entrepreneurship. This will in turn limit entrepreneurship and labor demand, supporting low wages and leading to multiple steady states (as I describe in the Appendix). In the next section, we will see that the interaction between parenting decisions and political behavior can lead to a more robust source of multiplicity.

\section{The Socio-Political Equilibrium}

In this section, I endogenize the key policy tool in this economy, the entry barrier, using a dynamic game of collective action. I then determine conditions under which there will be high entry barriers. I adopt a very simple political economy setup with a single non-standard feature. I suppose that obedient non-entrepreneurs may be convinced to vote against their economic interests.

Specifically, consider a situation in which non-entrepreneurs are a majority but there is already a high entry barrier. All else equal, non-entrepreneurs should be able to organize politically and push for policies that are more in line with their economic interests - in this instance, one involving zero entry barriers. However, one might imagine that obedient types are not only less willing to take unsanctioned actions in organizations by shirking, but they may also be less willing to create opposition against whatever political authority is dominant at the time. Put differently, obedient non-entrepreneurs may follow entrepreneurs' political preferences, in the same way that they obey them in the workplace (or may simply be less 
active in opposing the prevailing political equilibrium).

I introduce these considerations into the model analyzed so far in a reduced-form manner and suppose that with probability $\eta>0$ each obedient non-entrepreneur votes in a way that the entrepreneurs prefer (against their own economic interests).

Next note that independent entrepreneurs always prefer a prohibitively high entry barrier (say $\bar{b}$, though any entry barrier higher than this is equivalent). The preference of obedient entrepreneurs is more complex because they may prefer low entry barriers leading to high wages, so as to leave entrepreneurship and become workers. Nevertheless, we will see that they typically prefer high entry barriers, which induce low wages and thus create significant profit opportunities for entrepreneurs.

Let us now define $E^{*}$ as the equilibrium fraction of entrepreneurs when entry barriers are zero. This is given as a solution to the following equation (from the analysis leading up to Proposition 22:

$$
E^{*}\left(\frac{\varkappa}{w^{*}(0)}\right)^{\frac{1}{1-\alpha}} A_{i}^{\frac{1}{1-a}}=1-\bar{\mu}^{e}+\left(\bar{\mu}^{e}-E^{*}\right) \gamma
$$

where $w^{*}(\cdot)$ is again given by (16), and I have used the fact that in such an equilibrium all $1-\bar{\mu}^{e}$ of obedient types in the population (resulting from independent parenting style from all parents) will be in production work, and of the $\bar{\mu}^{e}$ independent types, $\bar{\mu}^{e}-E^{*}$ will be in production work. The equilibrium wage has to be $w^{*}(0)$, as in Proposition 2 , since otherwise all independent types would prefer to enter entrepreneurship.

Now the key question for the social-political equilibrium is whether in a steady state with $E^{*}$ entrepreneurs and a fraction $\bar{\mu}^{e}$ of independent types in the population, the political equilibrium will support zero entry barriers. A sufficient condition for this is

$$
E^{*}+\eta\left(1-\bar{\mu}^{e}\right)<(1-\eta)\left(1-\bar{\mu}^{e}\right)+\bar{\mu}^{e}-E^{*} .
$$

This condition ensures that in the candidate steady-state equilibrium where there is full employment and thus all parents choose independent values, there are sufficiently many independent non-entrepreneurs that they will be pushing for their own interests (despite the fraction $\eta$ of obedient non-entrepreneurs voting in line with entrepreneurial interests). In particular, existing entrepreneurs always prefer high entry barriers in order to increase their rents and will all vote for high entry barriers. They will also receive the vote of fraction $\eta$ of obedient workers. In this candidate steady-state equilibrium all entrepreneurs are independent, and thus all obedient types are workers. As a result, the total votes in favor of entrepreneurs is upper-bounded 
by the left-hand side of $(26), E^{*}+\eta\left(1-\bar{\mu}^{e}\right)$ (this is an upper bound, since $E^{*}$ is an upper bound on the number of existing entrepreneurs). Against this are all the votes of all independent agents who are not currently entrepreneurs and the remaining fraction $1-\eta$ of obedient workers. Independent workers always prefer low entry barriers, since $w^{*}(b)$ is diminishing in $b$, and independent non-entrepreneurs entering entrepreneurship always prefer low entry barriers as well. Hence, votes for low entry barriers are lower-bounded by $\bar{\mu}^{e}-E^{*}+(1-\eta)\left(1-\bar{\mu}^{e}\right)$, which gives us the right-hand side of (26).

Second, and in contrast to the previous case, consider a measure of entrepreneurship $E^{S}(\bar{b})$ given by

$$
E^{S}(\bar{b})\left(\frac{\varkappa}{\underline{w}_{i}}\right)^{\frac{1}{1-\alpha}}\left(\bar{\mu}^{e} A_{i}^{\frac{1}{1-a}}+\left(1-\bar{\mu}^{e}\right) A_{o}^{\frac{1}{1-\alpha}}\right) \in\left(E^{S}(\bar{b})\left(1-\bar{\mu}_{o}^{n}\right), E^{S}(\bar{b})\right) .
$$

This expression defines $E^{S}(\bar{b})$ as the measure of entrepreneurship, such that at wage $\underline{w}_{i}$ and when non-entrepreneurs are choosing obedient parenting, labor demand in steady state is enough to employ all obedient types plus no more than the fraction $1 / \gamma$ of the independent types (which gives $E^{S}(\bar{b})\left[\left(1-\bar{\mu}_{o}^{n}\right)+\frac{1}{\gamma} \times \gamma \times \bar{\mu}_{o}^{n}\right]=E^{S}(\bar{b})$ ). Because there is more than enough labor demand to employ all obedient workers, but not enough to employ all workers, the wage has to be at $\underline{w}_{i}$. Moreover, because no more than a fraction $1 / \gamma$ of independent types are finding employment, obedient parenting is a best response for non-entrepreneur parents. Notice also that the entry barrier here is $\bar{b}$, and hence there is no room for further entry into entrepreneurship (or any incentive for exit from entrepreneurship for obedient types). Finally, whether the entry barrier is $\bar{b}$ or in fact greater is not material, since any entry barrier $b \geq \bar{b}$ is equivalent in terms of preventing all entry.

Suppose, in addition, that the following condition is satisfied:

$$
E^{S}(\bar{b})+\eta\left(1-\bar{\mu}_{o}^{n}\right)\left(1-E^{S}(\bar{b})\right)>\left(1-\eta\left(1-\bar{\mu}_{o}^{n}\right)\right)\left(1-E^{S}(\bar{b})\right) .
$$

This condition implies that at the level of labor demand given by $E^{S}(\bar{b})$, there are sufficiently many obedient non-entrepreneurs and $\eta$ is sufficiently high that entrepreneurs' interests will win out in the political process. Specifically, in this case with high entry barriers, there is no entry into entrepreneurship, and hence all $E^{S}(\bar{b})$ entrepreneurs are naturally in favor of high entry barriers (the equilibrium wage they pay, $w^{*}(b)$, is decreasing in $b$ ). They will be joined with a fraction $\eta$ of obedient workers. The right-hand side, on the other hand, is the total votes of independent workers plus obedient workers who are not aligned with entrepreneurs (fraction $(1-\eta)$ ). This gives condition 28 ). 
This argument, together with the same reasoning that led to Proposition 2, establishes our main result in this section:

Proposition 3 Suppose that Assumptions 1-4 hold, and that for $E^{*}$ and $E^{S}(\bar{b})$ given by (25) and (27) conditions (26) and (28) are satisfied. Suppose also that $A_{o}>\underline{w}_{i}^{\alpha} A_{i}^{1-\alpha} / \gamma$. Then there exist multiple steady states.

- In one steady state, there are no entry barriers and this leads to an equilibrium measure of entrepreneurs given by $E^{*}$ as in (25). In addition, only independent types are entrepreneurs, the equilibrium wage is $w^{*}(0)$ and there is full employment. This encourages independent parenting among non-entrepreneurs, and given (26), generates sufficient political support to maintain zero entry barriers.

- In another steady state, entry barriers are given by $\bar{b}$ (or $b \geq \bar{b}$ ), the equilibrium measure of entrepreneurs is $E^{S}(\bar{b})$, there is no exit of obedient types from entrepreneurship, the equilibrium wage is $\underline{w}_{i}$, there is rationing of employment for independent types (with the probability of employment for any independent type worker being less than $1 / \gamma$ ), and non-entrepreneurs choose obedient parenting. Given (28), there is political support for high entry barriers.

First note that $E^{S}(\bar{b})$ that satisfies the conditions of the proposition is not necessarily unique, and hence there may be a range of values for $E^{S}(\bar{b})$ that can be supported as steadystate equilibria (and which one emerges will depend on initial conditions).

Much of the argument underlying the proof of this proposition was provided above, and here I sketch some of the remaining details. First note that $E^{*}$ is, by definition, the measure of entrepreneurs that will ensure full employment when the fraction of the population who are independent is $\bar{\mu}^{e}$ and the fraction of entrepreneurs who are independent is 1 . Then the equilibrium wage has to be $w^{*}(0)$ from Proposition 2. Because there is full employment, it follows immediately that independent parenting is optimal for all agents. To ensure that this is a steady-state equilibrium, all we need to check is that there is political support for zero entry barriers, which was established with the arguments leading to the proposition.

Next, a similar argument establishes that $E^{S}(\bar{b})$ is consistent with a different type of steadystate equilibrium. Now labor demand is sufficiently low that less than $1 / \gamma$ fraction of independent types can get jobs in the production sector. This encourages non-entrepreneurs to choose obedient parenting, and ensures that a smaller fraction of workers are independent. In 
particular, now in steady state only $\bar{\mu}^{n}<\bar{\mu}^{e}$ fraction of non-entrepreneurs are independent. Then, condition (28) ensures that there is enough support for high entry barriers, with the backing of a $\eta$ fraction of obedient non-entrepreneurs. The last condition we have to check to establish that this configuration is a steady-state equilibrium is that obedient entrepreneurs do indeed prefer high entry barriers in this case. This is straightforward to verify. If they exit entrepreneurship, the highest wage they can receive is $w^{*}(0)=A_{i} \gamma^{-(1-\alpha)}$ as given by (16) — if there is suddenly a jump to zero entry barriers. In contrast, in entrepreneurship they will make profits given by $A_{o}^{\frac{1}{1-\alpha}} \underline{w}_{i}^{-\frac{\alpha}{1-\alpha}}$. The condition in the proposition ensures that the latter is greater than the former, guaranteeing that obedient entrepreneurs always prefer entrepreneurship at low wages to production work at high wages (and this condition can be simultaneously satisfied with the Assumption 4).

What about the dynamic equilibrium in this economy? I now sketch the arguments that characterize the dynamic equilibrium. Suppose we start with $E_{0}$ entrepreneurs and fraction $\mu_{0}^{n}$ of non-entrepreneurs being independent. For simplicity, suppose first that $\mu_{0}^{n} \approx \bar{\mu}_{o}^{n}$. Then if

$$
E_{0}+\eta\left(1-\mu_{0}^{e}\right)\left(1-E_{0}\right)>\left(1-\eta\left(1-\mu_{0}^{e}\right)\right)\left(1-E_{0}\right)
$$

then there will again be a coalition in favor of high entry barriers. But once we impose entry barriers of $\bar{b}$ (or above), the probability of unemployment for independent types is greater than $1 / \gamma$, inducing non-entrepreneur parents to choose obedient parenting style. This reinforces the coalition in favor of high entry barriers, and the economy converges to one of the steady states with $E^{S}(\bar{b})$ entrepreneurs and high entry barriers. ${ }^{20}$ Suppose next that we start with an allocation where the opposite inequality in (29) holds and where, in addition, $\mu_{0}^{n} \approx \bar{\mu}^{e}$, then we will converge to the steady-state with $E^{*}$ entrepreneurs and low entry barriers ${ }^{21}$

The significance of this result is that we now have multiple steady states for the same parameters (though arising only for different initial conditions). These steady states have very different social hierarchies, distinct social mobility patterns and economic allocations, and critically, very different types of political engagement from non-elites (non-entrepreneurs). Two societies in such different steady states may appear to be trapped by their cultures, supporting

\footnotetext{
${ }^{20} \mathrm{We}$ can also see the role of the assumption that $\mu_{0}^{n} \approx \bar{\mu}_{o}^{n}$. If instead we had $\left.\mu_{0}^{n}<<\bar{\mu}_{o}^{n}, 29\right)$ might have been satisfied despite the fact that $E_{0}$ was quite high, and in this case, even after the obedient parenting decisions of non-entrepreneurs, the dynamics would ultimately lead to high labor demand and push the economy towards the zero entry barrier steady state.

${ }^{21}$ The role of the assumption that $\mu_{0}^{n} \approx \bar{\mu}^{e}$ is analogous to the argument provided in the previous footnote: if we had $\mu_{0}^{n}>>\bar{\mu}^{e}$, then even after independent parenting decisions by non-entrepreneurs, the coalition in favor of no entry barriers may not be sustainable.
} 
divergent political and economic outcomes. However, cultural configurations, as well as social hierarchies, mobility patterns and economic allocations, are all jointly determined. It is as much cultural configurations that are adjusting to different labor market equilibria as it is the other way around - labor markets and political behavior adapting to different cultures. Indeed, the source of multiplicity of steady states comes from the interplay of parental incentives to impart obedient values to their offspring and the political implications of a high fraction of obedient types. In particular, when wages are low and there is rationing of jobs, non-entrepreneurs prefer an obedient parenting style, and this then changes the collective action problem among workers, increasing the political power of entrepreneurs/elites and enabling them to introduce or maintain high entry barriers. High entry barriers then keep wages low, limit social mobility and maintain entrepreneur/elite privileges.

\section{Conclusion and Discussion}

There is growing interest in the role of cultural factors in shaping economic allocations. One of the most influential approaches within this domain has been to assume that cultural traits are slow-changing (perhaps determined by slow evolution or genetic or regional characteristics), which minimizes the impact of economic allocations on culture. This approach does not preclude an analysis of the co-evolution of culture and economic outcomes, for example as in Tabellini (2008) or Bisin and Verdier (2017), but in most applications, culture tends to have the more defining effect on economic allocations and institutions than the other way around. An alternative framework has recently been proposed in Acemoglu and Robinson (2021), emphasizing the fluidity of cultures and their adaptation to changing political or economic forces across societies. This approach puts more emphasis on the response of cultural variables to economic equilibria.

This paper explores how similar forces work across different social strata within a given society. Motivated by rich heterogeneities that exist in attitudes and social preferences within many societies, I study how labor market equilibria provide differential cultural incentives to individuals depending on their current social position, which in turn affects productivity, opportunities for entrepreneurship, and social mobility. My focus has been on the choice of values related to obedience vs. independence by families.

In the model, obedience is a useful characteristic for employers, especially when wages are low, since independent workers need to be given more incentives, whereas when wages are high, 
these incentives are automatically provided. Hence, in low-wage environments, low-income families will be pushed towards imparting values of obedience in order not to disadvantage their children in the labor market. To the extent that independence is useful for entrepreneurial activities, this then depresses their social mobility. High-income (entrepreneur) parents, on the other hand, always impart values of independence, since they expect that their children can enter into higher-income entrepreneurial (or managerial) activities thanks to their family resources and privileges, which is captured in this framework by their ability to stay in entrepreneurship without paying entry costs.

Social mobility dynamics and barriers to entrepreneurship play an important role in the model, because they determine economic opportunities, employment levels and wages. These in turn influence incentives for different parenting styles, which also play into economic opportunities and the extent of labor demand. Critically, however, the impacts of the labor market equilibrium are not the same on elite (entrepreneur) and non-elite (worker) parents. When labor demand is expected to be low, this encourages non-elite parents to choose very different values than elite parents, and this in turn further reduces social mobility.

The last section of the paper discussed how political activity can be hampered when labor market incentives encourage greater obedience. In particular, greater obedience at the workplace may translate into greater political obedience, bending the ark of political equilibrium in a more pro-elite direction. This creates a new source of multiplicity of steady-state equilibria: when entry barriers are high, labor demand is low and non-entrepreneur parents are encouraged to impart obedient values. Because obedient workers are less effective (and less consistent) in pursuing their economic and political interests, this can create a political equilibrium in which entry barriers can stay high and social mobility low. In contrast, when entry barriers are low, labor demand is high and non-entrepreneurs choose independent parenting. This increases the number of independent types among workers, who then become the backbone of a political coalition maintaining low entry barriers and high social mobility.

There are several interesting research areas highlighted by the analysis in this paper. To start with, there is need for more systematic empirical analysis of the relationship between labor market equilibrium and parental choices. The evidence presented in Figure 1 documents significant within-society heterogeneity in obedience versus independence, and suggests that there might be major interconnections, but does not speak to issues of causality.

There are also several theoretical and conceptual directions for future work. Here I discuss five. 
First, the paper assumed that obedience and independence are determined by parenting style. An alternative is to assume that these values are imparted by schools. Suppose for simplicity that parents have no influence on the values of their offspring, but these are determined by schools, and consider two different school structures. In the first, there is integrated schooling, so children of entrepreneurs and non-entrepreneurs go to the same schools. As a result, they will receive the same values. In the other, there is segregation across social class lines, and now the offspring of non-entrepreneurs could receive values of obedience. Suppose also that the same political economy structure as in the previous section determines school integration or segregation. Now, if entrepreneurs are sufficiently powerful but also sufficiently few so that they can significantly benefit from high entry barriers, then they may also have an incentive to create a segregated schooling structure in order to increase obedience among nonentrepreneurs. Particularly when employment is rationed, having more obedient workers in the workforce is beneficial for the entrepreneurs. This reasoning implies that it is indeed beneficial for current entrepreneurs to set up a segregated schooling structure (provided that this has no other costs). If, in contrast, society started with sufficiently powerful non-entrepreneurial interests, they would push for integrated schooling, and this would lead to a political equilibrium, supporting integrated schools and greater social mobility.

Second, one could consider an extension in which there is a within-family decision problem as well, whereby independent offspring may create lower utility for parents (because independent children are more likely to disobey their parents or less likely to contribute to within-family public goods). The main trade-off is that the economic gain from independence now needs to exceed a higher threshold for parents to be convinced to impart independent values to their children.

Third, it would be interesting to explore how the choice between obedience vs. independence affects political participation and social decisions in greater detail. Although this paper has assumed that obedient types may be uniformly more likely to go along with policy choices in line with elite interests, reality may be more complex. For example, obedient types may also be quite effective "foot soldiers" in a hierarchical party opposed to elite interests, or they may be inclined to support not the elite's interests but the status quo, thus amplifying persistence. More broadly, exploring the relationship between different types of values and priorities individuals receive from their parents and how these impact political activism is an interesting area for research.

Fourth, richer political economy dynamics can also be introduced into this framework. 
An important aspect would be the role of repression. Political repression may have different effects on obedient and independent workers, creating another set of economic and political interactions.

Finally, the framework in Acemoglu and Robinson (2021) emphasizes individual and collective choices about cultural configurations that take place at higher frequencies (and not simply during one's "impressionable years"). Incorporating these types of responses in the context of the interplay between obedience to hierarchy and labor market equilibrium is another interesting area of research. 


\section{Appendix A}

\section{Multiple Equilibria}

Let us next consider the same environment in the text, but with the extension mentioned in Remark 2, where non-entrepreneurs only have an opportunity to become an entrepreneur with probability $\lambda<1$. Let us also focus on the case in which $b<b^{*}$ in Proposition 2. The main difference from the model in the text is that now there can be multiple equilibria, particularly when $\pi_{o o}$ is low. The reasoning for the multiplicity is straightforward. When $\lambda<1$, an individual must take into account the likelihood that his offspring will not be able to enter into entrepreneurship even if he is of independent type. In this case, prevailing wages and labor market rationing become important. If there is rationing in equilibrium, non-entrepreneur parents might be discouraged from imparting independent values, but then when they choose obedient values, there will be less entry of high productivity individuals into entrepreneurship, keeping labor demand low, wages low and labor market rationing high, and this will in turn discourage an independent parenting style among non-entrepreneurs.

To simplify the exposition in this section, I focus on the extreme case in which $\pi_{o o}=0.22$ I also take the initial conditions $\mu_{0}^{n}=0$ and $\mu_{0}^{e}=\bar{\mu}^{e}$ (which is again adopted to simplify the discussion).

Consider first the case in which $\lambda$ is small. In this case, an independent offspring of a non-entrepreneur is unlikely to be able to enter into entrepreneurship. But the assumption that $\mu_{0}^{e}<\tilde{\mu}^{e}$ implies that at the initial date, there is rationing of jobs for independent workers, and anticipating this, it is preferable for non-entrepreneurs to choose obedient parenting styles. Since $\pi_{o o}=0$, this calculus of non-entrepreneur parents recurs in every period - i.e., if nonentrepreneur parents choose obedient parenting for their offspring at time $t$, then the next generation will also do likewise, and so there will be no further entry into entrepreneurship.

It is also straightforward to determine what the threshold value $\lambda^{*}$ above which this reasoning ceases to apply is. In particular, note that the worst scenario for an independent parenting style for non-entrepreneurs is one in which $q^{i}=0$. But even in this case, a nonentrepreneur may choose an independent parenting style with the hope of his offspring entering into entrepreneurship. It is straightforward to see that he will do so only if $\lambda>\lambda^{*}$, where $\lambda^{*}\left(\frac{A_{i}}{\underline{w}_{i}}\right)^{\frac{1}{1-\alpha}} \underline{w}_{i}=\underline{w}_{i}$ (the left-hand side is the expected income of an independent offspring at-

\footnotetext{
${ }^{22}$ When $\pi_{o o}$ is relatively high, even in cases where non-entrepreneur parents choose to impart obedient values, there will be sufficient independent types in the next generation, which may prevent the emergence of the low-wage, low-entrepreneurship equilibrium.
} 
tempting to enter into entrepreneurship, and the right-hand side is the income of an obedient offspring). This yields the critical value for $\lambda$ as:

$$
\lambda^{*}=\left(\frac{A_{i}}{\underline{w}_{i}}\right)^{-\frac{1}{1-\alpha}} .
$$

What happens when all non-entrepreneurs choose independent parenting? In this case, there will be some independent types among non-entrepreneurs in the next period, and a fraction $\lambda$ of these agents will find it profitable to enter entrepreneurship (since $b<b^{*}$ ). Then at the next date, $t=1$, the number of entrepreneurs and fraction of independent types among entrepreneurs are given by

$$
E_{1}=E_{0}+\lambda \pi_{o i}\left(1-E_{0}\right) \text { and } \mu_{1}^{e}=\frac{\bar{\mu}^{e} E_{0}+\lambda \pi_{o i}\left(1-E_{0}\right)}{E_{0}+\lambda \pi_{o i}\left(1-E_{0}\right)} .
$$

The intuition for these expressions is straightforward. With independent parenting among non-entrepreneurs and starting with no independent types among non-entrepreneurs, there will be a total of $\pi_{o i}\left(1-E_{0}\right)$ independent types among their offspring in the next period. A fraction $\lambda$ of those will have an opportunity to enter into entrepreneurship. This implies that the total number of entrepreneurs will increase from $E_{0}$ to $E_{0}+\lambda \pi_{o i}\left(1-E_{0}\right)$. Naturally, this will also increase the fraction of independent types among entrepreneurs from $\bar{\mu}^{e}$ to $\mu_{1}^{e}$. For independent parenting to be preferable for non-entrepreneurs, it is necessary that entry into entrepreneurship sufficiently increases the probability of employment of independent types . In particular, the analog of the condition in Claim 3 would be sufficient for this. This is equivalent to the condition in the proposition, namely $\lambda>\hat{\lambda}$ where

$$
\hat{\lambda}=\frac{1-E_{0}-E_{0}\left(\frac{\varkappa}{\underline{w}_{i}}\right)^{\frac{1}{1-\alpha}}\left[\bar{\mu}^{e} A_{i}^{\frac{1}{1-a}}+\left(1-\bar{\mu}^{e}\right) A_{o}^{\frac{1}{1-a}}\right]}{\pi_{o i}\left(1-E_{0}\right)\left[1+\left(\frac{\varkappa}{\underline{w}_{i}}\right)^{\frac{1}{1-\alpha}} A_{i}^{\frac{1}{1-a}}\right]} .
$$

As in (11), this expression requires that labor demand at time $t=1$ is exactly such that at the resulting probability of employment for independent workers, non-entrepreneur parents are indifferent between imparting independent and obedient values.

This discussion gives the main insight from this Appendix: when $\hat{\lambda}<\lambda^{*}$, there will be a multiplicity of equilibria. When sufficiently many non-entrepreneurs choose independent parenting, labor demand is high and this encourages other non-entrepreneurs to impart independent values to their offspring. However, when very few non-entrepreneurs choose independent parenting, labor demand is low and this discourages independent parenting, because parents 
are worried that their independent offspring will not be able to enter entrepreneurship, and when this happens, they will be rationed out of employment in favor of obedient types. Com-

paring $\mathrm{A}-1$ and $\mathrm{A}-2$, it is clear that when $A_{i}, \bar{\mu}^{e}$ or $\pi_{o i}$ is high, this inequality is likely to be satisfied.

\section{Data Sources and Additional Figures}

The data used in Figure 1, Figure 2 and in this Appendix are from the Integrated Values Survey, a series of nationally representative surveys covering 113 countries and around 432,463 respondents between 1981 and 2018. Interviews are conducted in the local languages and questions are designed to assess respondents' attitudes on a range of issues, including the three outcomes I look at in this paper: support for independence, obedience and hard work.

Independence, obedience and hard work are dummies that are equal to one if the respondent mentions these characteristics when asked: "Here is a list of qualities that children can be encouraged to learn at home. Which, if any, do you consider especially important?".

We also use two additional variables in our analysis. The first is family income, which is a self-reported measure based on the question "Here is a scale of incomes. We would like to know in what group your household is, counting all wages, salaries, pensions and other incomes that come in. Just give the letter of the group your household falls into, before taxes and other deductions." The scale goes from A to L (1 to 10 in the figure), representing the lowest and the highest values. The second is parent's education, which is based on the question "What is the highest level your father (mother) has completed in education?" (parent's education is based on the father, unless he did not live with the respondent at age 14, in which case, the survey inquires about the mother). This variable was only incorporated in the two final waves of the survey and it is therefore available for a smaller sample of 102,126 respondents.

In our sample, the proportion of respondents that consider independence, obedience and hard work as especially important are $48.06 \%, 34.78 \%$ and $52.80 \%$.

The following two figures present analogues of Figure 1, but also controlling for country fixed effects and survey wave fixed effects in the next figure, and additionally for year of birth, age and year of interview fixed effects in the following one. The results are very similar to those depicted in Figure 1, underscoring the within-country dimension of much of the variation in the data and the robustness of the relationship between independence vs. obedience and social economic status (and education) of parents. 
Figure A-1: Attitudes towards independence, obedience and hard work by family income and parents' education. This figure replicates Figure 1, but while controlling for fixed effects of country and survey wave. See the data and sample details below.
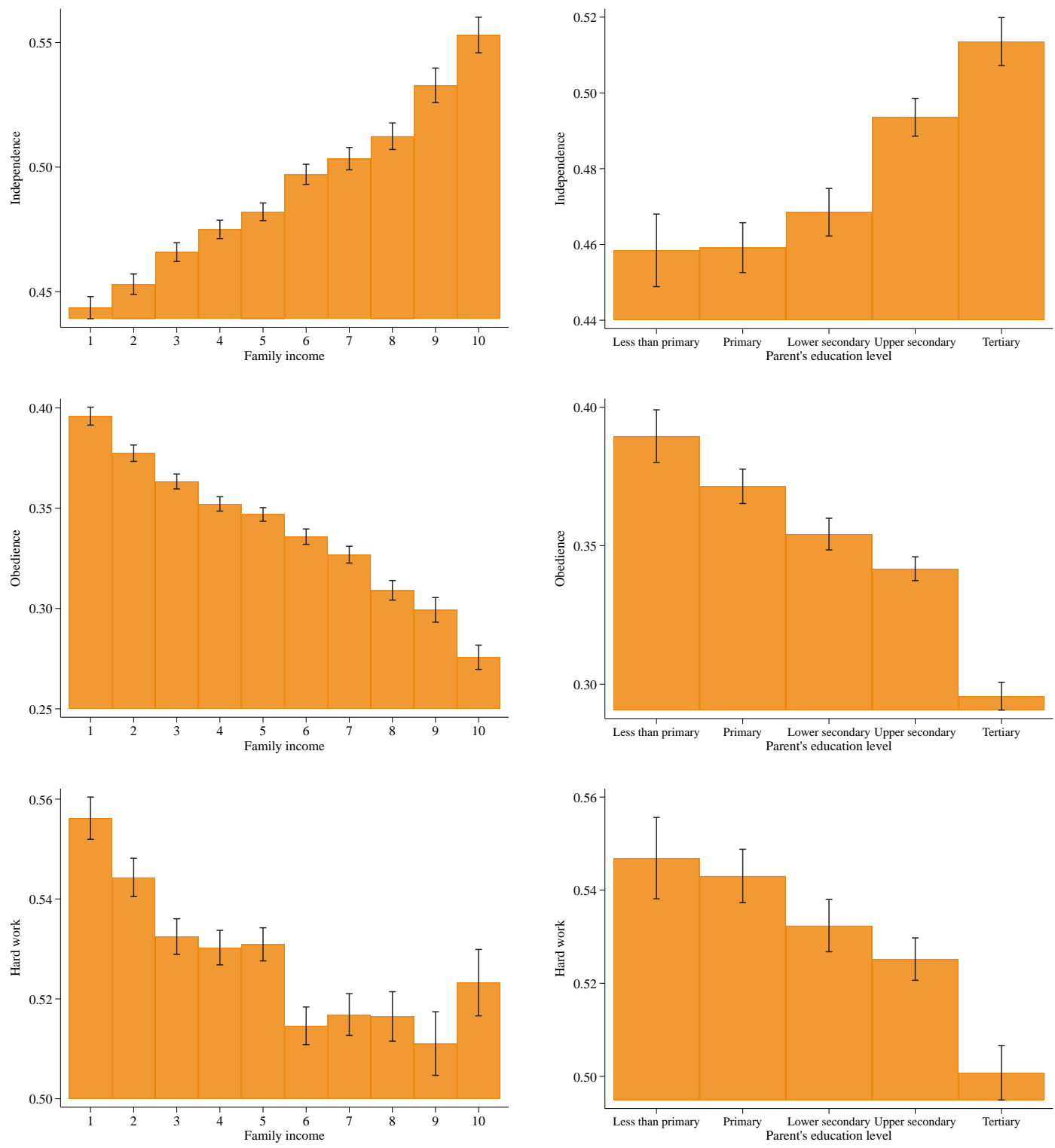

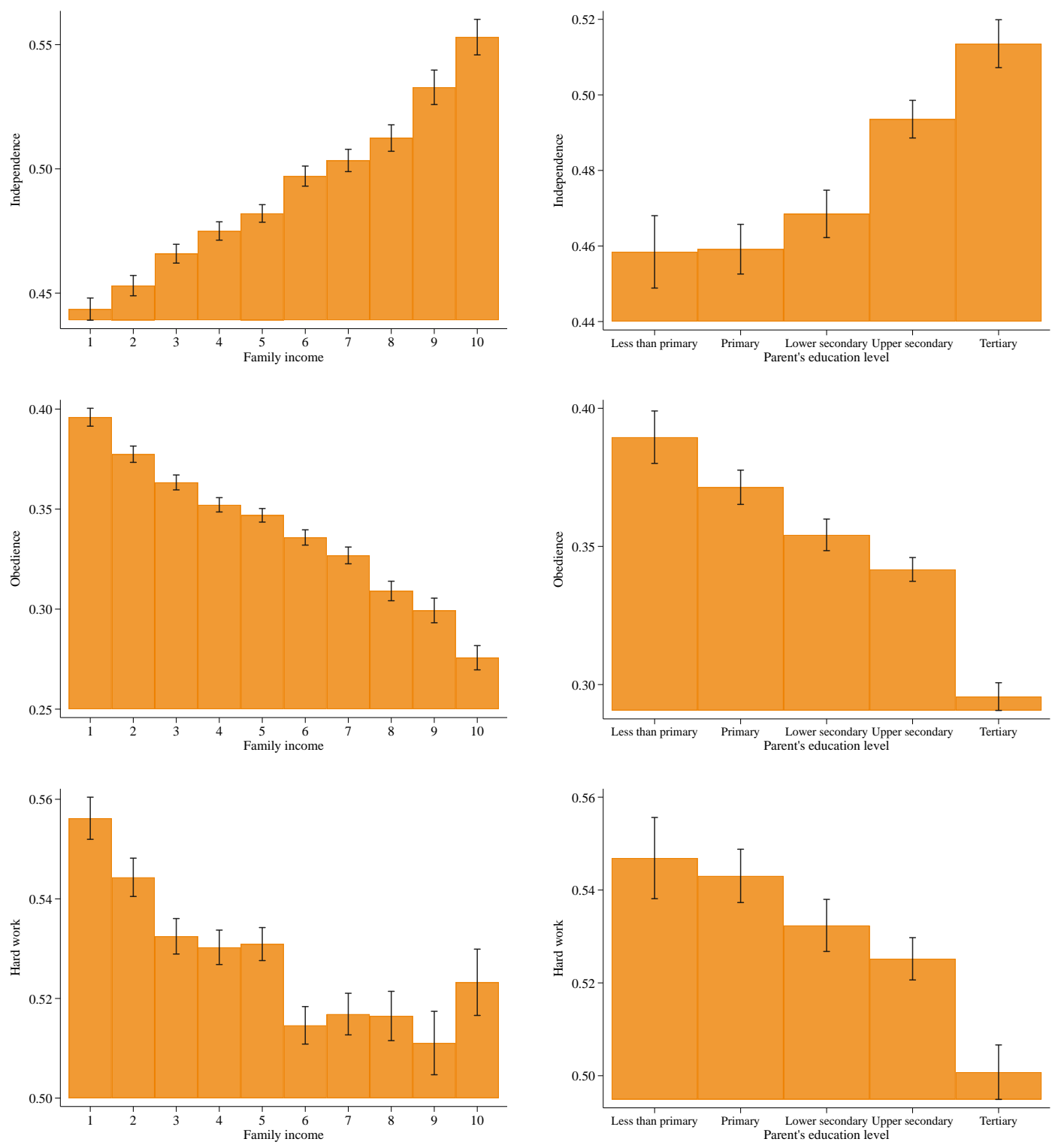

Figure A-2: Attitudes towards independence, obedience and hard work by family income and parents' education. This figure replicates Figure 1, but while controlling for fixed effects of country, survey wave, cohort, age and year of interview. See below for data and sample details. 


\section{References}

Acemoglu, Daron (2008) "Oligarchic vs. Democratic Societies" Journal of the European Economic Association 6(1), 1-44.

Acemoglu, Daron, Nicolas Ajzenman, Cevat Aksoy, Martin Fiszbein and Carlos Molina (2021) "Institutions Shape Social Preferences," mimeo.

Acemoglu, Daron, Georgy Egorov, and Konstantin Sonin (2018) "Social Mobility and Stability of Democracy: Re-evaluating De Tocqueville," Quarterly Journal of Economics, 133(2), 1041-1105.

Acemoglu, Daron and Matthew O. Jackson (2015) "History, Expectations, and Leadership in the Evolution of Social Norms," Review of Economic Studies, 82(1), 1-34.

Acemoglu, Daron and Matthew O. Jackson (2017) "Social Norms and the Enforcement of Laws." Journal of the European Economic Association, 15(2), 245-295.

Acemoglu, Daron and James A. Robinson (2021) "Institutions and Social Equilibria: A Framework," mimeo.

Acemoglu, Daron, Simon Johnson and James A. Robinson (2005) "Institutions as a Fundamental Cause of Growth," in Philippe Aghion and Steve Durlauf, eds. The Handbook of Economic Growth, Amsterdam, North Holland: El Sevier.

Alesina, Alberto, Paola Giuliano and Nathan Nunn (2013) "On the Origins of Gender Roles: Women and the Plough," Quarterly Journal of Economics, 128(2), 469-530.

Alesina, Alberto and Paola Giuliano (2015) "Culture and Institutions," Journal of Economic Literature, 53(4), 898-944.

Algan, Yann and Pierre Cahuc (2010) "Inherited Trust and Growth," American Economic Review, 100(5), 2060-2092.

Almond, Gabriel A., and Sidney Verba (1963) The Civic Culture: Political Attitudes and Democracy in Five Nations. Princeton, NJ: Princeton University Press.

Banfield, Edward (1958) The Moral Basis of a Backward Society. Glencoe, IL: The Free Press.

Benabou, Roland, and Efe Ok (2001) "Social Mobility and the Demand for Redistribution: The POUM Hypothesis," Quarterly Journal of Economics, 116(2), 447-487.

Benabou, Roland and Jean Tirole (2003) "Intrinsic and Extrinsic Motivation," Review of Economic Studies, 70(3), 489-520.

Besley, Timothy (2020) "State Capacity, Reciprocity, and the Social Contract," Econo- 
metrica, 88(4), 1307-1335.

Besley, Timothy and Torsten Persson (2019) "Democratic Values and Institutions," American Economic Review: Insights, 1(1), 59-76.

Bisin, Alberto, Jared Rubin, Avner Seror and Thierry Verdier (2021) "Culture, Institutions and the Long Divergence," NBER Working Paper No. 28488.

Bisin, Alberto and Thierry Verdier (2000) "Beyond the Melting Pot: Cultural Transmission, Marriage and the Evolution of Ethnic and Religious Traits," Quarterly Journal of Economics, 115(3), 955-988.

Bisin, Alberto and Thierry Verdier (2001) "The Economics of Cultural Transmission and the Dynamics of Preferences," Journal of Economic Theory, 97(2), 298-319.

Bisin, Alberto, Avner Seror and Thierry Verdier (2019). "Religious Legitimacy and the Joint Evolution of Culture and Institutions," in Jean-Paul Carvalho, Sriya Iyer and Jared Rubin, eds. Advances in the Economics of Religion, New York, NY: Palgrave Macmillan.

Bisin, Alberto and Theirry Verdier (2017) "On the Joint Evolution of Culture and Institutions," NBER Working Paper No. 23375.

Bourdieu, Pierre, and Jean-Claude Passeron (1990) Reproduction in Education, Society and Culture. New York, NY: Sage.

Bowles, Samuel, and Herbert Gintis (1976) Schooling in Capitalist America. New York, NY: Basic Books.

Angus Campbell, Philip E. Converse, Warren E. Miller and Donald E. Stokes (1960). The American Voter. Chicago, IL: University of Chicago Press.

Cavalli-Sforza, Luigi Luca and Marcus W. Feldman (1981) Cultural Transmission and Evolution: A Quantitative Approach. Princeton, NJ: Princeton University Press.

DiMaggio, Paul (1997) "Culture and Cognition," Annual Review of Sociology, 23, 263287.

Doepke, Matthias and Fabrizio Zilibotti (2008) "Occupational Choice and the Spirit of Capitalism," Quarterly Journal of Economics, 123(2), 747-793.

Doepke, Matthias and Fabrizio Zilibotti (2012) "Intergenerational Transmission of Risk Preferences, Entrepreneurship, and Growth," In 2012 Meeting Papers (No. 246). Society for Economic Dynamics.

Doepke, Matthias and Fabrizio Zilibotti (2019) Love, Money, and Parenting: How Economics Explains the Way We Raise Our Kids. Princeton, NJ: Princeton University Press.

Dohmen, Thomas, Armin Falk, David Huffman, and Uwe Sunde (2012) "The 
Intergenerational Transmission of Risk and Trust Attitudes," Review of Economic Studies, $79(2), 645-77$.

Durkheim, Emile (1983). The Division of Labor in Society. Chicago, IL: Free Press.

Erikson, Robert and John Goldthorpe (1992) The Constant Flux: A Study of Class Mobility in Industrial Societies. Oxford, UK: Clarendon Press.

Fernàdez, Raquel (2011) "Does Culture Matter?" in Jess Benhabib, Matthew O. Jackson and Alberto Bisin, eds. Handbook of Social Economics, Vol. 1A, Amsterdam, Holland: El Sevier.

Fernàdez, Raquel and Alessandra Fogli (2009) "Culture: An Empirical Investigation of Beliefs, Work, and Fertility," American Economic Journal: Macroeconomics, 1(1), 146-77.

Geertz, Clifford (1973). "Thick Description: Toward an Interpretive Theory of Culture," in Clifford Geertz, eds. The Interpretation of Culutres, New York, NY: Basic Books.

Giuliani, Paola (2007) "Living Arrangements in Western Europe: Does Cultural Origin Matter?," Journal of the European Economic Association, 5(5), 927-952.

Gorodnichenko, Yuri and Gerard Roland (2017) "Culture, Institutions and the Wealth of Nations," Review of Economics and Statistics, 99(3), 402-416.

Greif, Avner (1994) "Cultural Beliefs and the Organization of Society: A Historical and Theoretical Reflection on Collectivist and Individual Societies," Journal of Political Economy, 102(5), 912-950.

Greif, Avner (2006) Institutions and the Path to the Modern Economy: Lessons from Medieval Trade. New York, NY: Cambridge University Press.

Guiso, Luigi, Paola Sapienza and Luigi Zingales (2006) "Does Culture Affect Economic Outcomes?" Journal of Economic Perspectives, 20(2), 23-48.

Guiso, Luigi, Paola Sapienza and Luigi Zingales (2009) "Cultural Biases in Economic Exchange?" The Quarterly Journal of Economics, 124(3), 1095-1131.

Haines, Elizabeth L. and John T.Jost (2000) "Placating the Powerless: Effects of Legitimate and Illegitimate Explanation on Affect, Memory, and Stereotyping." Social Justice Research, 13(3), 219-236.

Henrich, Joseph (2020) The WEIRDest People in ther World. New York, NY: Farrar, Straus and Giroux.

Hofstede, Geert. (1980). "Motivation, Leadership, and Organization: Do American Theories Apply Abroad?" Organizational Dynamics, 9(1), 42-63.

Ichino, Andrea and Giovanni Maggi (2000) "Work Environment and Individual Back- 
ground: Explaining Regional Shirking Differentials in a Large Italian Firm," Quarterly Journal of Economics, 115(3), 1057-90.

Inkeles, Alex (1960) "Industrial Man: The Relation of Status to Experience, Perception, and Value." American Journal of Sociology, 66(1), 1-31.

Jennings, M. Kent, Laura Stoker and Jake Bowers (2009) "Politics across Generations: Family Transmission Reexamined", The Journal of Politics, 71(3), 782-799.

Jost, John T. and Brenda Major (Eds.). (2001). The Psychology of Legitimacy: Emerging Perspectives on Ideology, Justice, and Intergroup Relations. Cambridge, UK: Cambridge University Press.

Jost, John T., Brett W. Pelham and Mauricio R. Carvallo (2002). "Non-conscious Forms of System Justification: Implicit and Behavioral Preferences for Higher Status Groups," Journal of Experimental Social Psychology, 38(6), 586-602.

Kohn, Melvin (1959). "Social Class and Parental Values," American Journal of Sociology, 64(4), 337-351.

Kohn, Melvin (1989). Class and Conformity: A Study in Values. Chicago, IL: University of Chicago Press.

Leventoğlu, Bahar (2005) "Social Mobility and Political Transitions," Journal of Theoretical Politics, 17(4): 465-496.

Lipset, Seymour Martin (1959) "Some Social Requisites of Democracy: Economic Development and Political Legitimacy," American Political Science Review, 53(1), 69-105.

Lipset, Seymour Martin (1963) Political Man. New York, NY: Doubleday.

Miller, Warren E. and J, Merrill Shanks (1996) The New American Voter. Cambridge, MA: Harvard University Press.

Miller, Carol T. and Cheryl R. Kaiser (2001) "A Theoretical Perspective on Coping with Stigma," Journal of Social Issues, 57(1), 73-92.

Mokyr, Joel (1990) The Lever of Riches. New York, NY: Oxford University Press.

Mokyr, Joel (2005) "The Intellectual Origins of Modern Economic Growth," Journal of Economic History, 65(2), 285-351.

Mokyr, Joel (2016) A Culture of Growth: The Origins of the Modern Economy. Princeton, NJ: Princeton University Press.

Negandhi, Anant R. and S. B. Prasad (1971) Comparative Management. New York, NY: Appleton-Century-Crofts.

Nie, Norman H., Sidney Verba and John Petrocik (1976) The Changing American 
Voter. Cambridge, MA: Harvard University Press.

Oakes, Jeannie (2005) Keeping Track: How Schools Structure Inequality. New Haven, CT: Yale University Press.

Parsons, Talcott (1951) The Social System. New York, NY: Free Press.

Patterson, Orlando (2014) "Making Sense of Culture," Annual Review of Sociology, 40, $1-30$.

Pearlin, Leonard I. and Melvin L. Kohn (1966) "Social Class, Occupation, and Parental Values: A Cross-national Stud," American Sociological Review, 31(4), 466-479.

Persson, Torsten and Guido Tabellini (2009). "Democratic Capital: The Nexus of Political and Economic Change," American Economic Journal: Macroeconomics, 1(2), 88-126.

Piketty, Thomas (1995) "Social Mobility and Redistributive Politics," Quarterly Journal of Economics, 110(3), 551-583.

Putnam, Robert H. (1993) Making Democracy Work, Princeton, NJ: Princeton University Press.

Putnam, Robert H. (2000) Bowling Alone. New York, NY: Simon and Schuster.

Roland, Gèrard (2004). "Understanding Institutional Change: Fast-moving and Slowmoving Institutions." Studies in Comparative International Development, 38(4), 109-131.

Rubin, Jared (2017) Rulers, Religion, and Riches Why the West Got Rich and the Middle East Did Not. New York, NY: Cambridge University Press.

Sewell, William (2005) Logics of History: Social Theory and Social Transformation. Chicago, IL: University of Chicago Press.

Spalaore, Enrico and Romain Wacziarg (2009) "The Diffusion of Development," Quarterly Journal of Economics, 124(2), 469-529.

Swidler, Ann (1986) "Culture in Action: Symbols and Strategies," American Sociological Review, 51(2), 273-286.

Swidler, Ann (2003) Talk of Love: How Culture Matters, Chicago, IL: University of Chicago Press.

Tabellini, Guido (2008) "The Scope of Cooperation: Values and Incentives," Quarterly Journal of Economics, 123(3), 905-950.

Tabellini, Guido (2010) "Culture and Institutions: Economic Development in the Explore Regions of Europe," Journal of the European Economic Association, 8(4), 677-816.

Ventura, Raphael (2001). "Family Political Socialization in Multiparty Systems." Comparative Political Studies, 34(6), 666-691. 
Willis, Paul (1981). Learning to Labor: How Working Class Kids Get Working Class Jobs. New York, NY:Columbia University Press. 